\title{
The histone deacetylase SIRT6 controls embryonic stem cell fate via TET-mediated production of 5-hydroxymethylcytosine
}

\author{
Jean-Pierre Etchegaray ${ }^{1,2,10}$, Lukas Chavez ${ }^{3,8,9}$, Yun Huang ${ }^{3,8,9}$, Kenneth N. Ross ${ }^{1,2}$, Jiho Choi ${ }^{1,2}$,
} Barbara Martinez-Pastor ${ }^{1,2}$, Ryan M. Walsh ${ }^{1,2}$, Cesar A. Sommer ${ }^{4}$, Matthias Lienhard ${ }^{3}$, Adrianne Gladden ${ }^{5}$, Sita Kugel ${ }^{1,2}$, Dafne M. Silberman ${ }^{6}$, Sridhar Ramaswamy ${ }^{1,2}$, Gustavo Mostoslavsky ${ }^{4}$, Konrad Hochedlinger ${ }^{1,2,7}$, Alon Goren ${ }^{5,10}$, Anjana Rao ${ }^{3}$ and Raul Mostoslavsky ${ }^{1,2,10}$

How embryonic stem cells (ESCs) commit to specific cell lineages and yield all cell types of a fully formed organism remains a major question. ESC differentiation is accompanied by large-scale histone and DNA modifications, but the relations between these epigenetic categories are not understood. Here we demonstrate the interplay between the histone deacetylase sirtuin 6 (SIRT6) and the ten-eleven translocation enzymes (TETs). SIRT6 targets acetylated histone H3 at Lys 9 and 56 (H3K9ac and H3K56ac), while TETs convert 5-methylcytosine into 5-hydroxymethylcytosine (5hmC). ESCs derived from Sirt6 knockout (S6KO) mice are skewed towards neuroectoderm development. This phenotype involves derepression of OCT4, SOX2 and NANOG, which causes an upregulation of TET-dependent production of $5 \mathrm{hmC}$. Genome-wide analysis revealed neural genes marked with $5 \mathrm{hmC}$ in S6KO ESCs, thereby implicating TET enzymes in the neuroectoderm-skewed differentiation phenotype. We demonstrate that SIRT6 functions as a chromatin regulator safeguarding the balance between pluripotency and differentiation through Tet-mediated production of $5 \mathrm{hmC}$.

During early stages of development, ESCs proliferate and differentiate into all somatic cell types. ESC differentiation requires global changes of chromatin architecture to elicit specific epigenetic programs of gene expression associated with each somatic cell type. Chromatin alterations including changes in histone modifications and DNA methylation patterns play a critical role during the commitment, establishment and maintenance of a particular cell lineage during early embryogenesis ${ }^{1}$. Notably, the interplay between these chromatin alterations, and how they execute epigenetic programs of gene expression during ESC differentiation remain largely unknown.

DNA methylation is usually linked to chromatin compaction and gene inactivation, which constitutes a critical process to establish cell lineage specification during ESC differentiation ${ }^{2}$. DNA methylation is a reversible process catalysed by the $\mathrm{Fe}^{2+}-$ and $\alpha$-ketoglutarate-dependent dioxygenases, TET enzymes ${ }^{3,4}$. There are three TET orthologues in the mouse, TET1, TET2 and TET3. These enzymes revert the methylation status of DNA by successive oxidation of 5-methylcytosine $(5 \mathrm{mC})$ into $5 \mathrm{hmC}, 5$-carboxycytosine $(5 \mathrm{caC})$ and 5 -formylcytosine $(5 \mathrm{fC})$, which are intermediates of an active DNA demethylation mechanism ${ }^{5,6}$. Increased levels of $5 \mathrm{hmC}$ are tightly associated with the maintenance of the pluripotency state of ESCs (refs 7-9). The expression of TET1 and TET2, maintained at high levels in ESCs, diminishes during differentiation, which correlates with repression of pluripotent genes and activation of developmental genes $^{4,10-13}$. The TET-dependent production of $5 \mathrm{hmC}$ has been implicated in cell lineage specification of ESCs (ref. 14). However, upstream regulatory mechanisms underlying the participation of TET enzymes and the potential role of $5 \mathrm{hmC}$ as a direct epigenetic

\footnotetext{
${ }^{1}$ The Massachusetts General Hospital Cancer Center, Harvard Medical School, Boston, Massachusetts 02114, USA. ${ }^{2}$ The MGH Center for Regenerative Medicine, Harvard Medical School, Boston, Massachusetts 02114, USA. ${ }^{3}$ La Jolla Institute for Allergy and Immunology, Sanford Consortium for Regenerative Medicine, UCSD Department of Pharmacology, UCSD Moores Cancer Center, La Jolla, California 92037, USA. ${ }^{4}$ The Center for Regenerative Medicine (CReM), Boston Medical Center, Boston University School of Medicine, Boston, Massachusetts 02118, USA. ${ }^{5}$ Broad Technology Labs (BTL), The Broad Institute of Harvard and MIT, Cambridge, Massachusetts 02142, USA. ${ }^{6}$ Department of Human Biochemistry, Medical School, CEFyBO-UBA-CONICET, Buenos Aires, CP1121, Argentina. ${ }^{7}$ Howard Hughes Medical Institute, Chevy Chase, Maryland 20815, USA. ${ }^{8}$ Present addresses: Division of Pediatric Neurooncology, German Cancer Research Center (DKFZ), Im Neuenheimer Feld 280, Heidelberg 69120, Germany (L.C.); Institute of Biosciences \& Technology, Texas A\&M University Health Science Center, Houston, Texas 77030, USA (Y.H.). ${ }^{9}$ These authors contributed equally to this work.

${ }^{10}$ Correspondence should be addressed to J-P.E., A.Goren or R.M.

(e-mail: etchegaray.jean-pierre@mgh.harvard.edu or agoren@broadinstitute.org or rmostoslavsky@mgh.harvard.edu)
}

Received 20 May 2014; accepted 3 March 2015; published online 27 April 2015; DOI: 10.1038/ncb3147 
component regulating specific genes during ESC differentiation remain undetermined.

One of the histone modifications involved in ESC function is acetylation of Lys 56 in histone H3 (H3K56ac), which has been linked to the pluripotent transcriptional network in human ESCs (ref. 15). More specifically, H3K56ac levels correlate with the transcriptional activation of pluripotent genes, its levels diminishing significantly on those genes during ESC differentiation ${ }^{15}$. How this mark is regulated during ESC differentiation remained unclear. The NAD-dependent histone deacetylase Sirt6 was shown to target H3K56ac in mouse ESCs (refs 16,17) and is one of seven mammalian members of the sirtuin protein network, with roles in genome stability, glucose metabolism and tumour suppression ${ }^{16-21}$. Owing to its ability to specifically target $\mathrm{H} 3 \mathrm{~K} 56 \mathrm{ac}$, we investigated the potential participation of SIRT6 in ESC differentiation. Our results demonstrate that SIRT6 directly regulates the expression of the core pluripotent genes Oct4, Sox 2 and Nanog, via deacetylation of $\mathrm{H} 3 \mathrm{~K} 56 \mathrm{ac}$, which in turn controls ESC differentiation through Tet-mediated oxidation of $5 \mathrm{mC}$ into $5 \mathrm{hmC}$.

\section{RESULTS}

\section{SIRT6 deletion skews ESC differentiation potential towards neuroectoderm}

ESCs derived from S6KO mouse embryos showed skewed differentiation compared with those derived from their wildtype WT littermates. When cultured to form embryoid bodies (EBs), S6KO EBs from three different ESC lines were significantly smaller in size compared with their WT counterparts (Fig. 1a). Immunofluorescence analysis showed expression of the endoderm marker GATA4 to be downregulated in S6KO EBs, while expression of the neuroectoderm marker GFAP was upregulated (Fig. 1b). GFAP is also upregulated in EBs derived from S6KO induced pluripotent stem cells (iPSCs; Supplementary Fig. 1A). The skewing of S6KO EBs towards neuroectoderm and away from endoderm, mesoderm and trophectoderm was confirmed by examining the expression of additional markers (Fig. 1c and Supplementary Fig. 1B). Moreover, when we subjected ESCs to an in vitro neurogenesis protocol, we found a striking increase in the number of nestinand $\beta$-III tubulin-expressing neurons in S6KO versus WT controls (Fig. 1d and Supplementary Fig. 1C,D). The expression of nestin was upregulated in S6KO EBs even under normal culturing conditions (Supplementary Fig. 1E). Notably, even before differentiation, S6KO ESCs exhibited a downregulation of genes associated with endoderm, mesoderm and trophectoderm, while neuroectoderm-related genes were upregulated, consistent with a primed differentiation state in the absence of SIRT6 (Fig. 1e and Supplementary Fig. 1F). These results point towards a previously unidentified role for Sirt6 in regulating cell lineage specification during ESC differentiation.

\section{Pluripotent genes are not repressed during differentiation of ESCs lacking SIRT6}

Persistent expression of Oct4, Sox 2 and Nanog is critical to maintain the pluripotency state, but it needs to be silenced on ESC differentiation ${ }^{22}$. However, during early stages of ESC differentiation, OCT4 and SOX2 were shown to orchestrate germ layer fate decisions. OCT4 was found to suppress neuroectoderm differentiation while promoting development of the mesoderm. Divergently, SOX2 inhibits mesodermal differentiation, but promotes the development of neuroectoderm. An overall downregulation of Nanog is a causal event to impel the differentiation state ${ }^{23}$. Thus, we assessed whether lack of Sirt6 could alter expression of these pluripotent genes following differentiation. Notably, Sirt6 deficiency was characterized by persistent expression of the core pluripotency genes Oct4, Sox2 and Nanog and their protein products in both ESCs and EBs (Fig. 1f-i). Using an alternative differentiation protocol (retinoic acid, RA) we find that even though OCT4 was normally repressed, the expression of SOX2 and NANOG persisted on forced differentiation (Supplementary Fig. 1G). These data suggest that Sirt6 negatively regulates the expression of these core pluripotent genes to achieve proper ESC differentiation.

\section{SIRT6 regulates levels of $\mathrm{H} 3 \mathrm{~K} 56 \mathrm{ac}$ and $\mathrm{H} 3 \mathrm{~K} 9 \mathrm{ac}$ at the promoters of Oct4, Sox2 and Nanog}

To determine the mechanism by which SIRT6 regulates expression of the core pluripotent genes, we subjected ESCs from both S6KO and WT to chromatin immunoprecipitation (ChIP) before and after differentiation. We first assessed binding of SIRT6 to the pluripotent genes. Notably, SIRT6 was found at the promoter regions of Oct4, Sox 2 and Nanog both before and after differentiation (Fig. 2a). We then tested for the presence of H3K56Ac, one of the SIRT6 substrates previously linked to pluripotent gene expression ${ }^{15}$. Consistently, the levels of $\mathrm{H} 3 \mathrm{~K} 56 \mathrm{ac}$ at these promoters were increased in $\mathrm{S} 6 \mathrm{KO}$ compared with WT ESCs and EBs (Fig. 2b). Additionally, another SIRT6 substrate, H3K9ac, was also increased at the promoter regions of these core pluripotent genes in S6KO versus WT ESCs (Fig. 2c). Furthermore, the recruitment of SIRT6 was extended inside the Oct4 locus, showing maximum binding at the promoter (primers $\mathrm{A}$ and $\mathrm{B}$ at -195 and -13 positions, respectively) and towards the $3^{\prime}$ end of exon 1 (primer $\mathrm{C}$ at position +719 ) before and after differentiation (Fig. 2d,e), which is paralleled by an increase in H3K56ac in S6KO versus WT EBs (Fig. 2f). The recruitment of Sirt6 was not apparent at position +4220 (primer D, between exons 4 and 5) towards the end of Oct4 paralleling the unchanged levels of H3K56ac between WT and S6KO EBs. Moreover, Sirt6-dependent deacetylation of both H3K9ac and H3K56ac on the Oct4, Sox2 and Nanog loci was confirmed by genome-wide analyses (Fig. 2g,h). Notably, an increase in these histone modifications is retained after RA-dependent differentiation, particularly at the Sox 2 locus (Supplementary Fig. 1H). These results support the idea that Sirt6 negatively regulates expression of the core pluripotency genes Oct4, Sox2 and Nanog, thereby emphasizing a critical role for Sirt6 in stem cell function. The inability to suppress these core pluripotent genes might in part account for the skewed differentiation towards the neural lineage in S6KO compared with WT ESCs and iPSCs.

\section{Global increase of TET expression and $5 \mathrm{hmC}$ in S6KO versus WT ESCS}

Tet 1 and Tet 2 genes are postulated targets of OCT4 and SOX2 (refs 14,24). Strikingly, we observed significant upregulation of Tet1 and Tet2 expression in S6KO compared with WT ESCs, at both messenger RNA and protein levels (Fig. 3a,b). Slot blot analysis showed a striking global increase of $5 \mathrm{hmC}$ in $\mathrm{S6KO}$ compared with WT ESCs, without a global alteration in the levels of $5 \mathrm{mC}$ (Fig. $3 \mathrm{c}, \mathrm{d}$ ). 


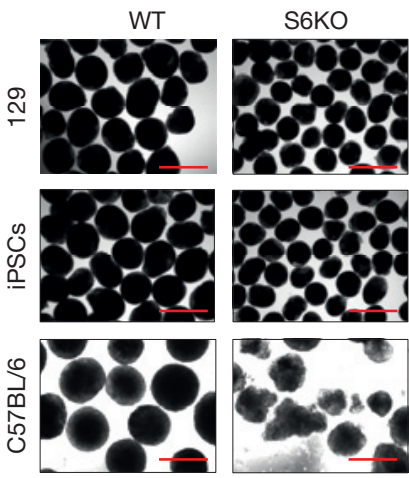

b
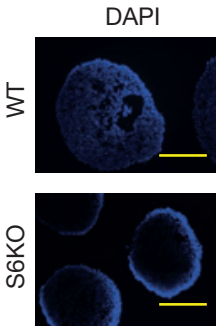

DAPI
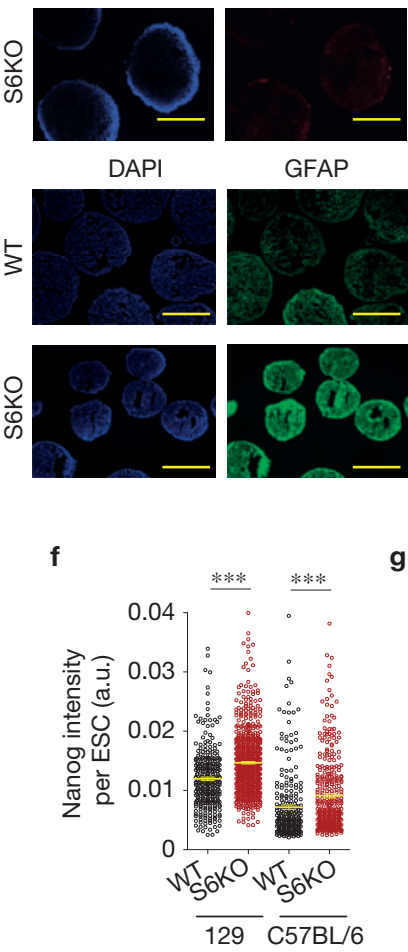

GFAP
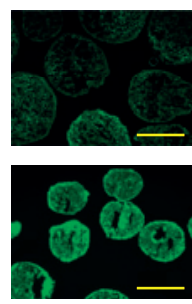
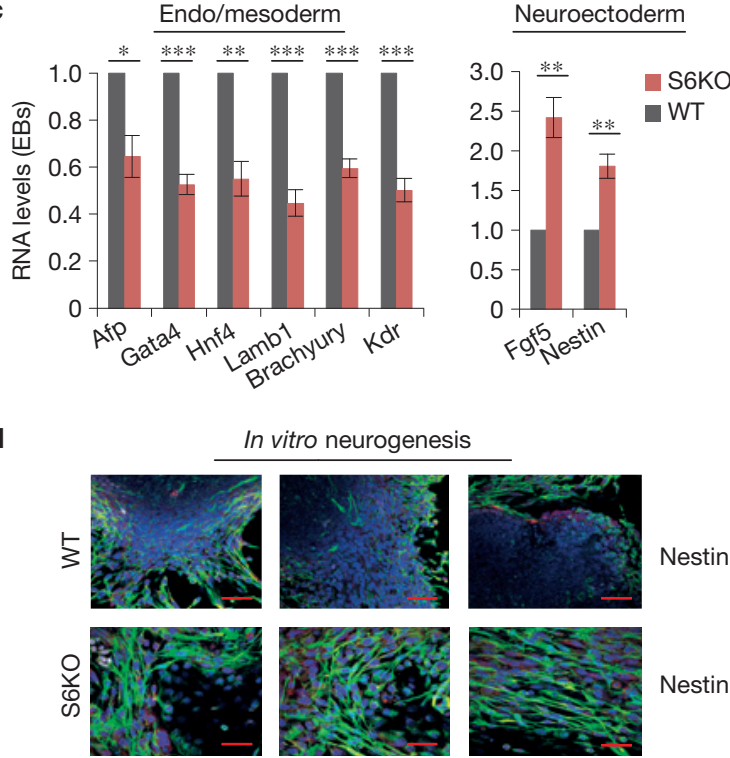

e
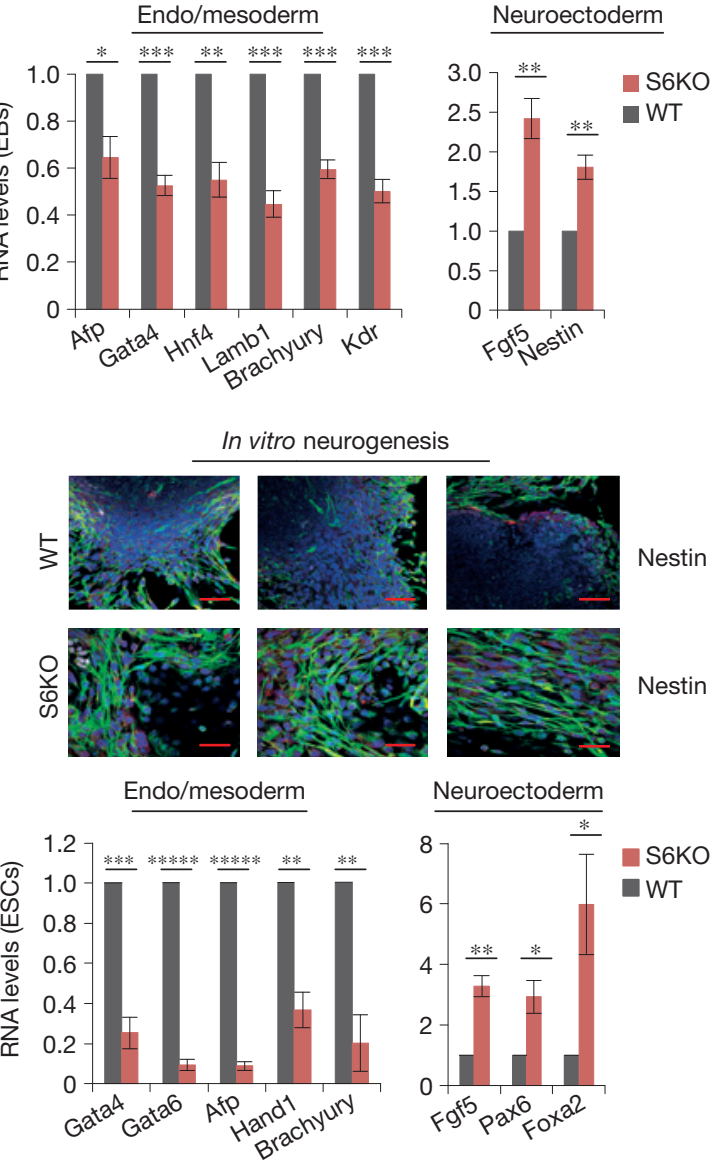

Nestin

stin g

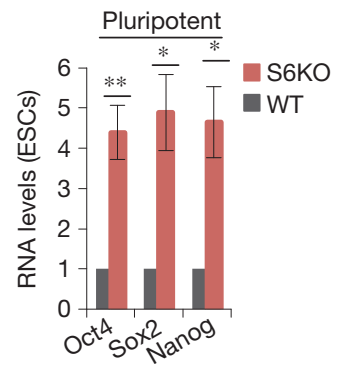

h

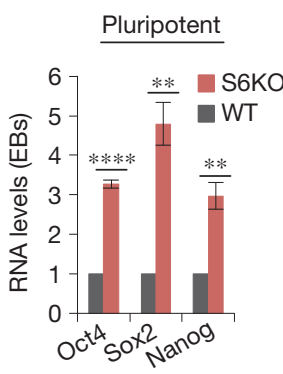

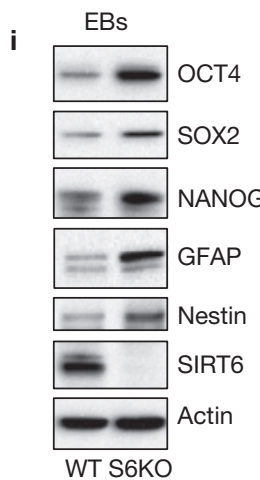

Figure 1 SIRT6 deficiency skews ESC differentiation towards neuroectoderm and promotes expression of Oct4, Sox2 and Nanog. (a) EBs derived from WT and S6KO ESCs (129, iPSCs from 129 and C57BL/6 mouse strain). Scale bars, $250 \mu \mathrm{m}$. (b) Immunofluorescence of EBs from WT and S6KO (129 mouse strain) for Gata4 (scale bars, $250 \mu \mathrm{m}$ ) and Gfap (scale bars, $500 \mu \mathrm{m}$ ). (c) Expression of endoderm, mesoderm and neuroectoderm genes in WT versus S6KO EBs. Quantitative PCR with reverse transcription (qRTPCR) data are expressed relative to WT EBs. (d) Immunofluorescence of in vitro-generated neurons from WT and S6KO EBs for nestin (green). Nuclei were stained with DAPI. Scale bars, $100 \mu \mathrm{m}$. (e) Expression of endoderm, mesoderm and neuroectoderm genes in WT versus S6KO ESCs. qRT-PCR data are expressed relative to WT EBs. (f) Quantification of Nanog levels

Concordantly to the upregulation of Tet genes in S6KO, we found an increased recruitment of OCT4 and SOX2 to both Tet 1 and Tet2 genes in S6KO versus WT ESCs by ChIP analysis, using primers for (mean intensity, a.u.) per cell by immunostaining in WT and S6KO ESCs from 129 and C57BL/6 genetic backgrounds. Yellow bars represent mean \pm s.e.m. ${ }^{* * *} P<0.001$ by one-way ANOVA followed by Tukey test analysis (a.u., arbitrary units). (g) Core pluripotent gene expression in WT versus S6KO ESCs assessed by qRT-PCR analysis. (h) Core pluripotent gene expression in WT versus S6KO EBs assessed by qRT-PCR analysis. (i) Western blot analysis for the core pluripotent factors on WT versus S6KO EBs. These are representatives of at least $n=3$ experimental replicates. The data in $\mathbf{c}, \mathbf{e}-\mathbf{h}$ are at least $n=3$ experimental replicates, values are mean \pm s.e.m. ${ }^{*} P<0.05,{ }^{* *} P<0.01$, ${ }^{* * *} P<0.001,{ }^{* * * *} P<0.0001,{ }^{* * * * *} P<0.00001$, by $t$-test analysis. qRT-PCR analysis in $\mathbf{c}, \mathbf{e}, \mathbf{g}, \mathbf{h}$ is from independent RNA preparations. Uncropped images of blots are shown in Supplementary Fig. 9.

previously identified OCT4:SOX2 consensus binding sites ${ }^{14}$ (Fig. 3e,f). Furthermore, levels of OCT4 and SOX2 binding to their own genes were enhanced in S6KO versus WT ESCs (Fig. 3g). These data 


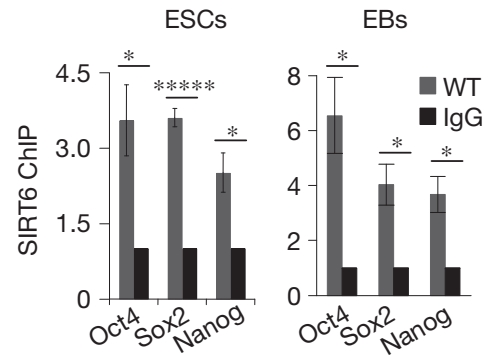

b

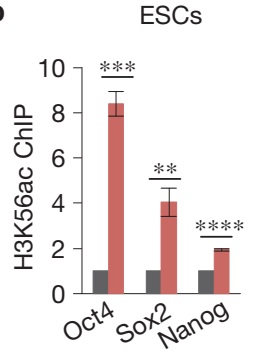

EBs

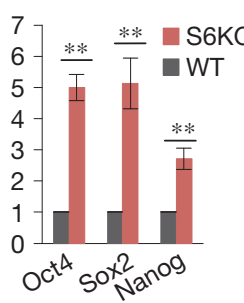

C

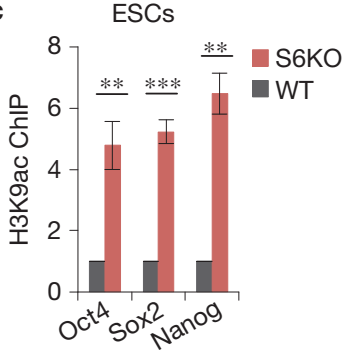

d

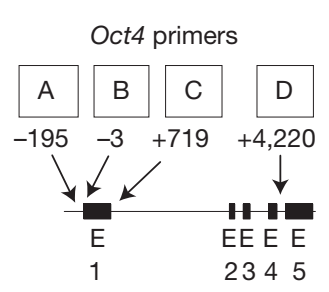

e

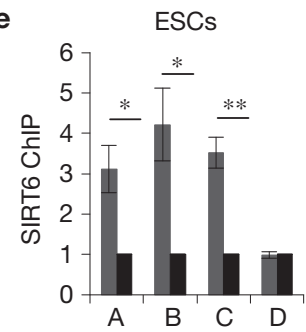

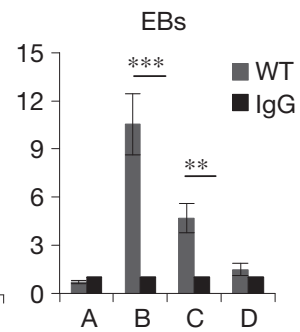

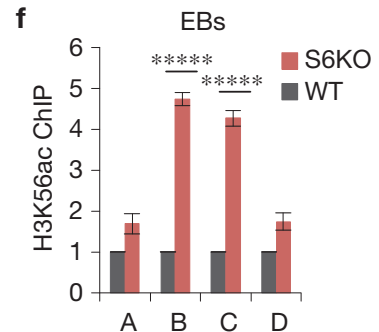

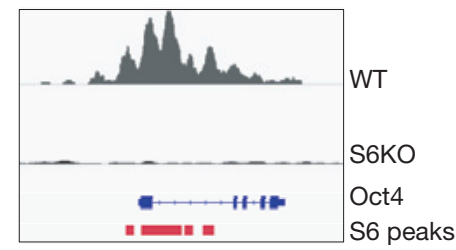

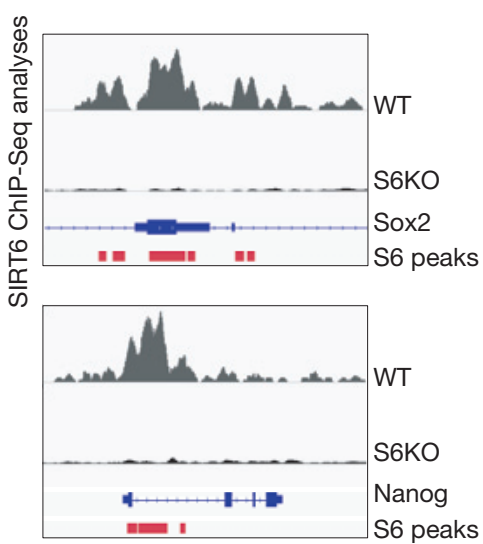

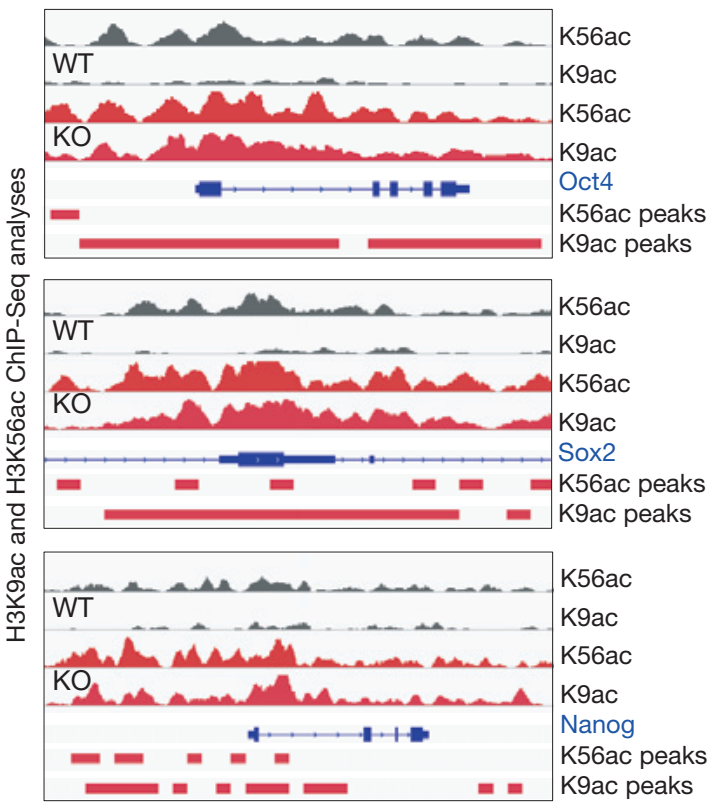

Figure 2 SIRT6-dependent regulation of core pluripotent genes. (a) ChIP analysis for Sirt6 on core pluripotent gene promoters in WT ESCs and EBs. Data are expressed relative to IgG ChIP control. (b) ChIP analysis for H3K56ac on core pluripotent gene promoters in both ESCs and EBs from WT and S6KO. Data are expressed relative to WT values. (c) ChIP analysis for H3K9ac on core pluripotent gene promoters in EBs from WT and S6KO. Data are expressed relative to WT values. (d) Schematic diagram of the Oct4 locus depicting primers used for ChIP assays in e,f. (e) ChIP analysis for SIRT6 on the Oct4 locus in WT ESCs and EBs. Data are expressed relative to IgG ChIP control. (f) ChIP analysis for H3K56ac on the Oct4 locus in EBs from WT and S6KO. Data are expressed relative to WT values. (g) SIRT6 ChIP-Seq binding profiles

support a positive role for OCT4:SOX2-dependent regulation of Tet expression, which is enhanced in S6KO compared with WT ESCs, and thereby suggest an important function for SIRT6, as a potential modulator of a Tet-dependent mechanism associated with ESC differentiation. on Oct4, Sox2 and Nanog genes in WT and S6KO ESCs. Images were created with the Integrative Genomic Viewer ${ }^{51}$. Data are normalized to total counts, and the scale range is 0.0-7.0. (h) ChIP-Seq binding profiles of histone marks H3K56ac and H3K9ac on Oct4, Sox2 and Nanog genes in WT and S6KO ESCs. Images were created with the Integrative Genomic Viewer ${ }^{51}$. Data are normalized to total counts, and the scale range is $0.0-2.0$. The red bars under each plot in $\mathbf{g}, \mathbf{h}$ represent statistically significant peaks for each ChIP-Seq analysis. The data in $\mathbf{a}-\mathbf{c}, \mathbf{e}, \mathbf{f}$ are $n=3$ experimental replicates (independent chromatin samples from different cell preparations); values are mean \pm s.e.m. ${ }^{*} P<0.05,{ }^{* *} P<0.01,{ }^{* * *} P<0.001,{ }^{* * * *} P<0.0001$, ${ }^{* * * * *} P<0.00001$, by $t$-test analysis.

\section{Depletion of TET1 or TET2 rescues the differentiation phenotype in S6KO ESCs}

The upregulation of TETs and $5 \mathrm{hmC}$ production in S6KO led us to examine the genetic interaction between SIRT6 and TETs in ESCs. Strikingly, short hairpin RNA (shRNA)-mediated depletion of 
a

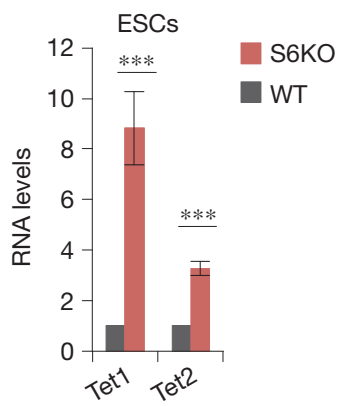

b

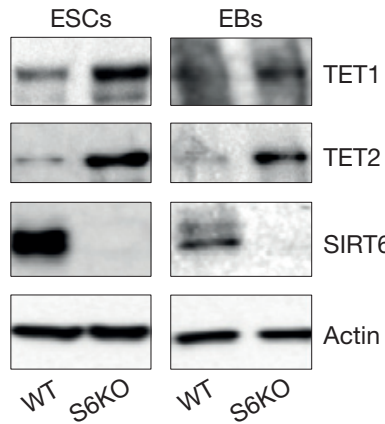

C

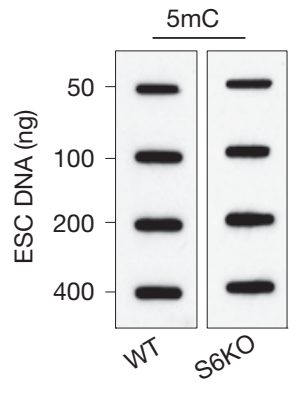

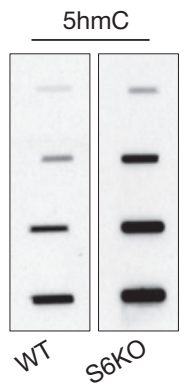

d

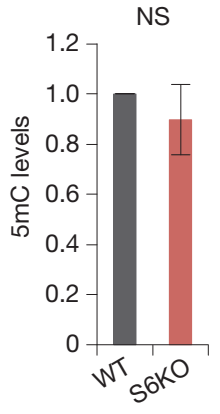

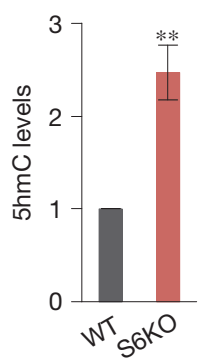

e

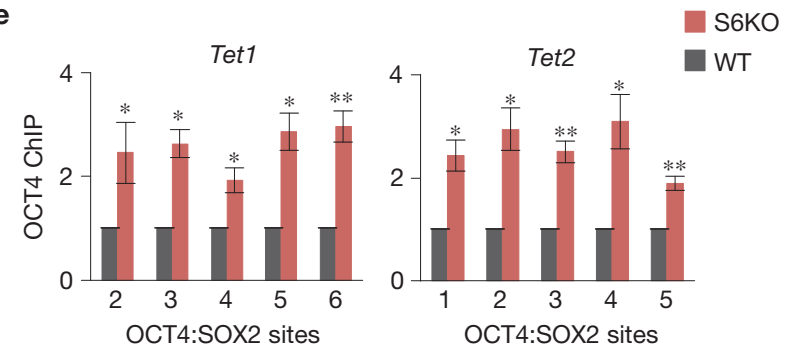

f

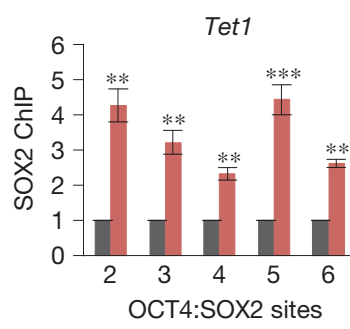

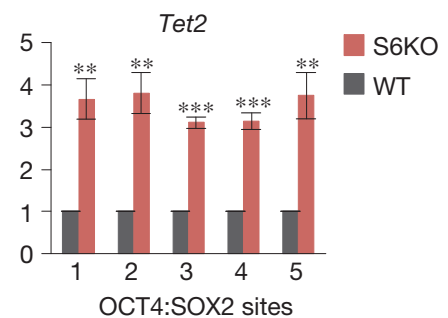

g

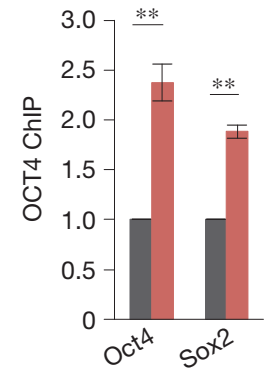

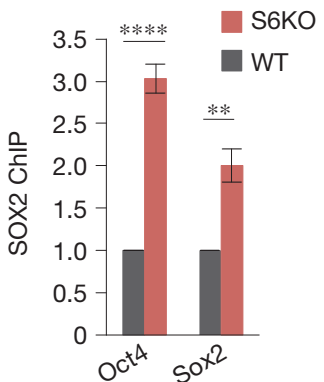

Figure 3 OCT4:SOX2-dependent upregulation of TETs in S6KO versus WT ESCs and EBs. (a) Tet1 and Tet2 gene expression in WT versus S6KO ESCs. qRT-PCR data are expressed relative to WT ESCs. Data are from $n=3$ experimental replicates (independent RNA preparations) and values are mean \pm s.e.m. ${ }^{* * *} P<0.001$, by $t$-test analysis. (b) Western blot analysis for TET1 and TET2 in both ESCs and EBs. A representative of $n=3$ biological replicates is shown. (c) Global $5 \mathrm{mC}$ and $5 \mathrm{hmC}$ levels assayed by slot blot analysis in WT versus S6KO ESCs. (d) Graphs showing fold change of $5 \mathrm{mC}$ and $5 \mathrm{hmC}$ from h. (e) ChIP analysis for OCT4 on both Tet1 and Tet2 at OCT4:SOX2 predicted binding sites ${ }^{14}$ in WT versus S6KO ESCs. Data are expressed relative to WT values. (f) ChIP analysis for SOX2 on both Tet1 and Tet2 at OCT4:SOX2 predicted binding sites ${ }^{14}$ in WT versus S6KO ESCs. Data are expressed relative to WT values. The data are $n=3$ experimental replicates. Values are mean \pm s.e.m. ${ }^{*} P<0.05,{ }^{* *} P<0.01,{ }^{* * *} P<0.001$, by $t$-test analysis. (g) ChIP analysis for OCT4 or SOX2 on both Oct4 and Sox2 genes in WT versus S6KO ESCs. Data are expressed relative to WT values. The data in $\mathbf{d}-\mathbf{g}$ are $n=3$ experimental replicates (independent chromatin samples from different cell preparations). Values are mean \pm s.e.m. ${ }^{*} P<$ $0.05,{ }^{* *} P<0.01,{ }^{* * *} P<0.001,{ }^{* * * *} P<0.0001$, by $t$-test analysis. NS, not significant. Uncropped images of blots are shown in Supplementary Fig. 9. either Tet1 or Tet2 (ref. 14) fully rescued not only the abnormal morphology of EBs derived from S6KO ESCs (Fig. 4a), but also the skewed differentiation, as demonstrated by the normalized expression of the neuroectoderm marker GFAP (Fig. 4b,c). Furthermore, the expression of additional germ layer markers-the neuroectoderm markers Fgf5 and nestin and the endoderm markers Gata4 and Gata6 - was restored following Tet knockdown (Fig. 4d). As expected, the levels of Tet 1 and Tet 2 transcripts, as well as Oct4 and Nanog transcripts, were brought back to near WT levels on Tet knockdown in S6KO EBs (Fig. 4e), as were the elevated levels of $5 \mathrm{hmC}$ in S6KO ESCs (Fig. 4f,g). Interestingly, knockdown of either Tet1 or Tet 2 caused downregulation of both Tet genes (Fig. 4e), thereby implicating both TETs in the S6KO differentiation phenotype. These results further support a role for SIRT6 in suppressing expression of the core pluripotent genes in both pluripotent and differentiating ESCs, thus indirectly controlling the levels of TET enzymes to facilitate proper differentiation.

\section{SIRT6 controls levels of $5 \mathrm{hmC}$ of genes associated with neuroectoderm}

To elucidate the role of TET proteins in WT and S6KO ESCs, we analysed the genome-wide distribution of $5 \mathrm{hmC}$ in WT and $\mathrm{S} 6 \mathrm{KO}$ ESCs by cytosine 5-methylenesulphonate immunoprecipitation ${ }^{25}$. We then compared $5 \mathrm{hmC}$ levels in adjacent, non-overlapping 500-basepair (bp) windows genome-wide ${ }^{26}$. S6KO ESCs showed significant $\left(P<1 \times 10^{-3}\right)$ gain of $5 \mathrm{hmC}$ at more windows $(2,218)$ than lost $5 \mathrm{hmC}$ 


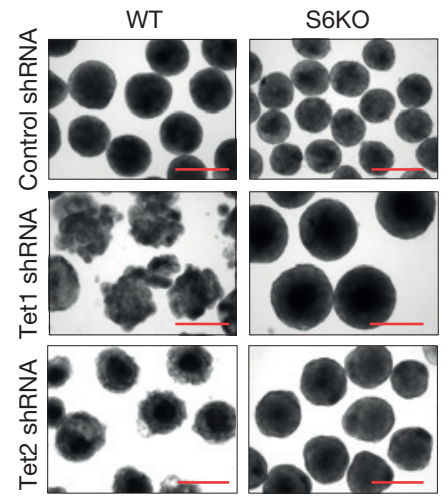

b

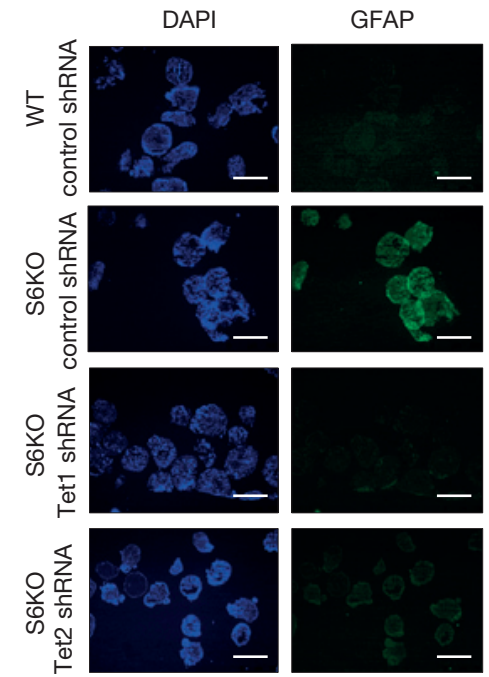

c

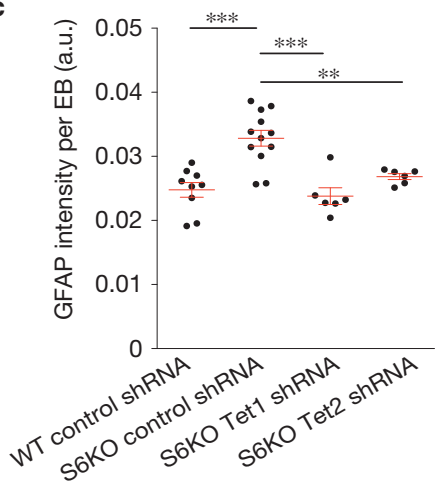

d

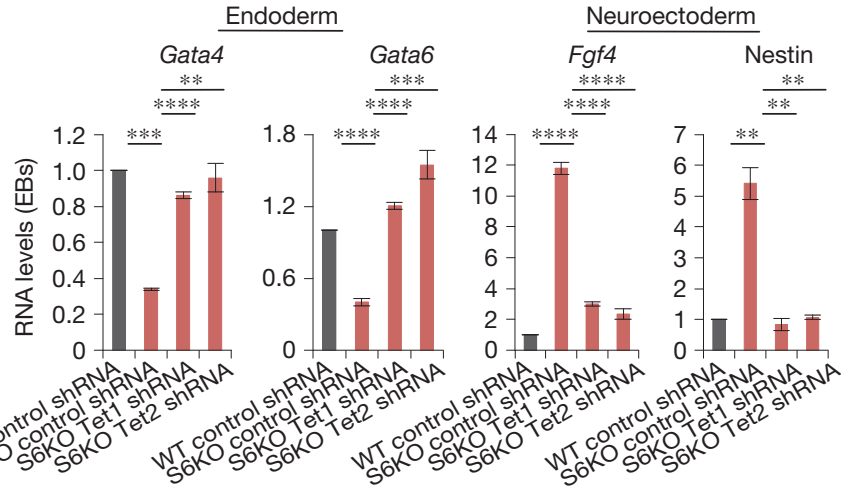

e
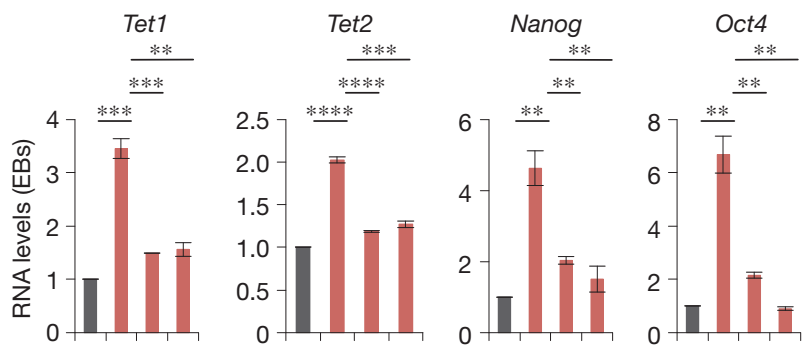

bntantontant

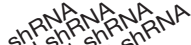

$0 T_{1}^{1}$
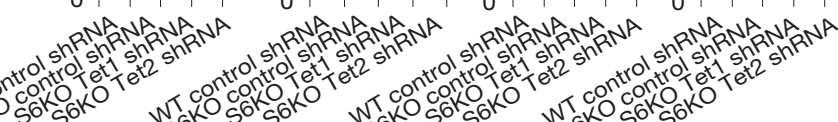

\section{f}

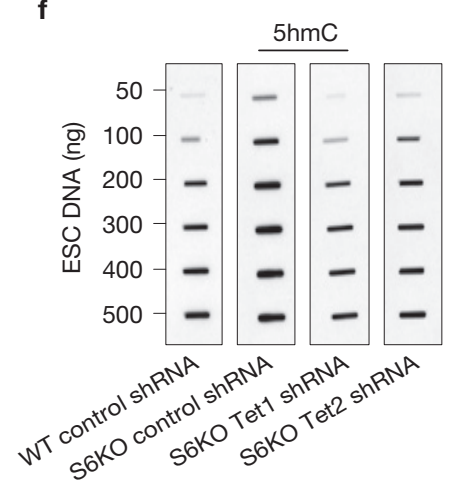

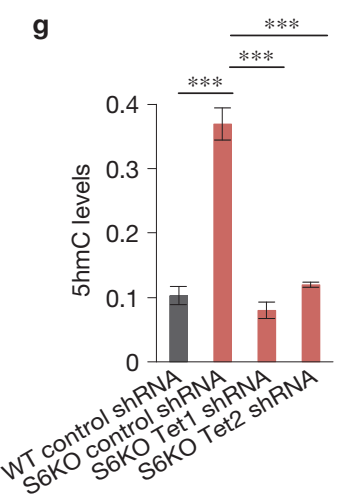

Figure 4 Tet knockdown rescues the differentiation phenotype of S6KO ESCs and global levels of 5hmC. (a) EBs derived from WT and S6KO ESCs stably infected with shRNA targeting Tet1 or Tet2. Scale bars, $500 \mu \mathrm{m}$. (b) Immunofluorescence of Tet knockdown EBs for GFAP. Scale bars, $500 \mu \mathrm{m}$. (c) Graph showing quantification of GFAP intensity (mean intensity, a.u.) per EB in $\mathbf{b}$. Data are represented as detection of GFAP per EB ( $n=3$ EB preparations from each genotype). Red bars represent mean \pm s.e.m. ${ }^{* *} P<0.01,{ }^{* * *} P<0.001$ by one-way ANOVA followed by Tukey test analysis. (a.u., arbitrary units). (d) Gene expression of endoderm, and neuroectoderm genes in Tet knockdown EBs. qRT-PCR data are expressed relative to WT EBs stably transfected with shRNA control. (e) Expression of Tet and core pluripotent genes in Tet knockdown EBs analysed as described above. (f) Global $5 \mathrm{hmC}$ levels assayed by slot blot analysis in Tet knockdown ESCs. (g) Graphs show fold change of $5 \mathrm{hmC}$ from $\mathbf{f}$. The data are represented as $n=3$ experimental replicates (independent DNA preparations from different cell cultures). Values are mean \pm s.e.m. ${ }_{* * *} P<0.001$, by $t$-test analysis. The data in $\mathbf{d}, \mathbf{e}$ and $\mathbf{g}$ are $n=3$ experimental replicates (qRT-PCR analysis from independent RNA preparations). Values are mean \pm s.e.m. ${ }^{*} P<0.05$, ${ }^{* *} P<0.01,{ }^{* * *} P<0.001,{ }^{* * * *} P<0.0001$, by $t$-test analysis. qRT-PCR analysis on $\mathbf{d}, \mathbf{e}$ are from independent RNA preparations. Uncropped images of blots are shown in Supplementary Fig. 9.
(1,562 windows; Supplementary Table 1$)$, confirming at a genomewide level the overall gain of $5 \mathrm{hmC}$ observed by dot blot analysis (Fig. 3c,d). Differentially hydroxymethylated regions (DHMRs) with gain of $5 \mathrm{hmC}$ were enriched over DHMRs with loss of $5 \mathrm{hmC}$ at promoters $\left(P<1.3 \times 10^{-252}\right.$ and $P<6.3 \times 10^{-16}$, respectively $)$ as well as $\mathrm{CpG}$ islands $\left(P<1.4 \times 10^{-269}\right.$ and $P<1.2 \times 10^{-48}$, respectively; 


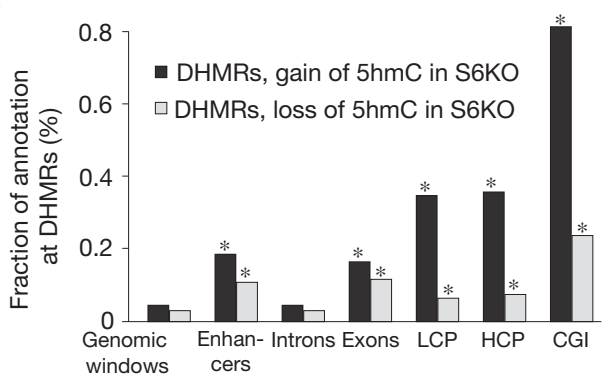

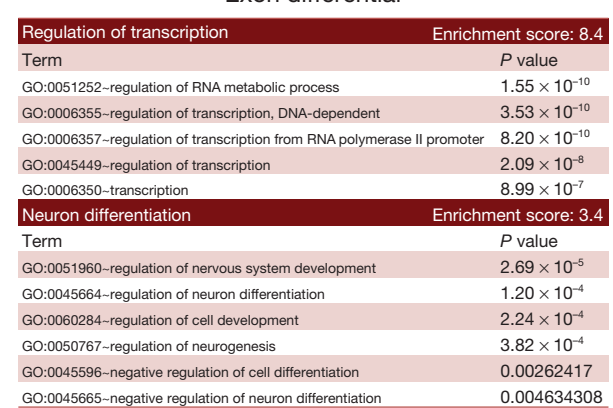

c

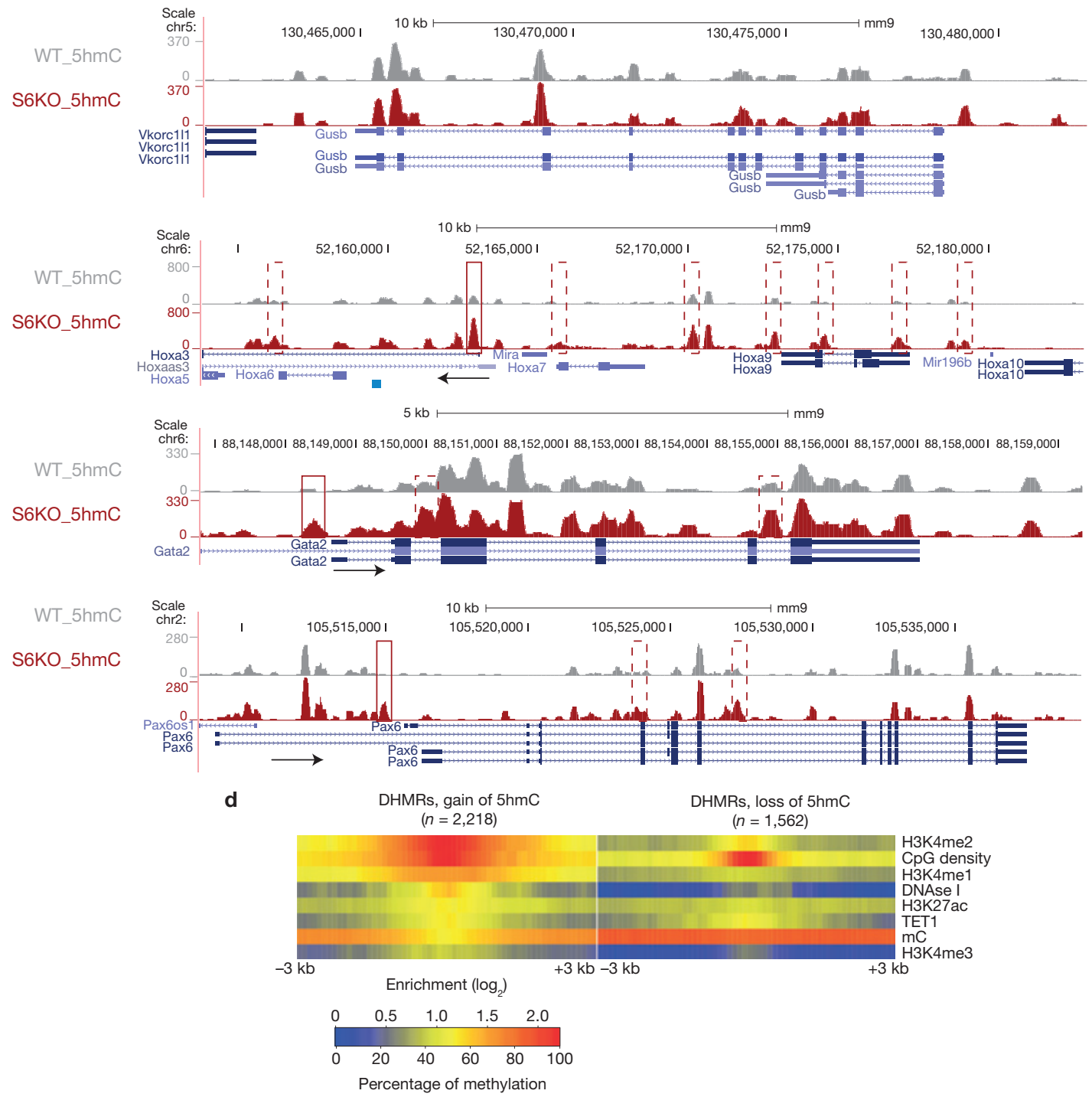

Figure 5 Characterization of genomic regions with change of $5 \mathrm{hmC}$ in $\mathrm{S} 6 \mathrm{KO}$ compared with WT ESCs. (a) Total DHMRs with gain $(n=2,218$ genomic windows) or loss ( $n=1,562$ genomic windows) of $5 \mathrm{hmC}$ in S6KO represent $0.04 \%$ or $0.03 \%$ of the genome, respectively (genomic windows). Both classes of DHMRs are significantly enriched at enhancers, exons, LCPs HCPs, and CpG islands ( $P$ value $\leq 1 \times 10^{-3}$, Fisher's exact test), where DHMRs with gain or loss of $5 \mathrm{hmC}$ are similarly enriched at exons ( $P$ value $\leq 6.3 \times 10^{-155}$ and $P$ value $\leq 1.5 \times 10^{-110}$, respectively), whereas DHMRs with gain of $5 \mathrm{hmC}$ are much stronger enriched than DHMRs with loss of $5 \mathrm{hmC}$ at promoters (LCPs and HCPs, $P$ value $\leq 1.3 \times 10^{-252}$ and $P$ value $\leq 6.3 \times 10^{-16}$, respectively), and at $\mathrm{CpG}$ islands ( $P$ value $\leq 1.4 \times 10^{-269}$ and $P$ value $\leq 1.2 \times 10^{-48}$, respectively). (b) Functional annotation ${ }^{45}$ of genes with change of $5 \mathrm{hmC}$ (gain or loss) at exons reveals significantly enriched gene ontology clusters associated with regulation of transcription and neuron differentiation. (c) UCSC browser visualization of gain of $5 \mathrm{hmC}$ in S6KO (red) versus WT (grey) at the promoter of Hoxa3, Gata2 and Pax6 genes, and at multiple other genomic regions in the vicinity. No changes in $5 \mathrm{hmC}$ levels on the Gusb gene, is shown as an analytical control. (d) Enrichment analysis of histone $\mathrm{H} 3$ modifications strongly connects H3K4me2 and low-methylated regions to DHMRs with gain of $5 \mathrm{hmC}$ in S6KO. Both, gain and loss of $5 \mathrm{hmC}$ occurs at regions with elevated levels of $\mathrm{CpG}$ density and TET1 binding. The marks are sorted by high (top) to low (bottom) enrichment at the centre of DHMRs with gain of $5 \mathrm{hmC}$ in S6KO. 


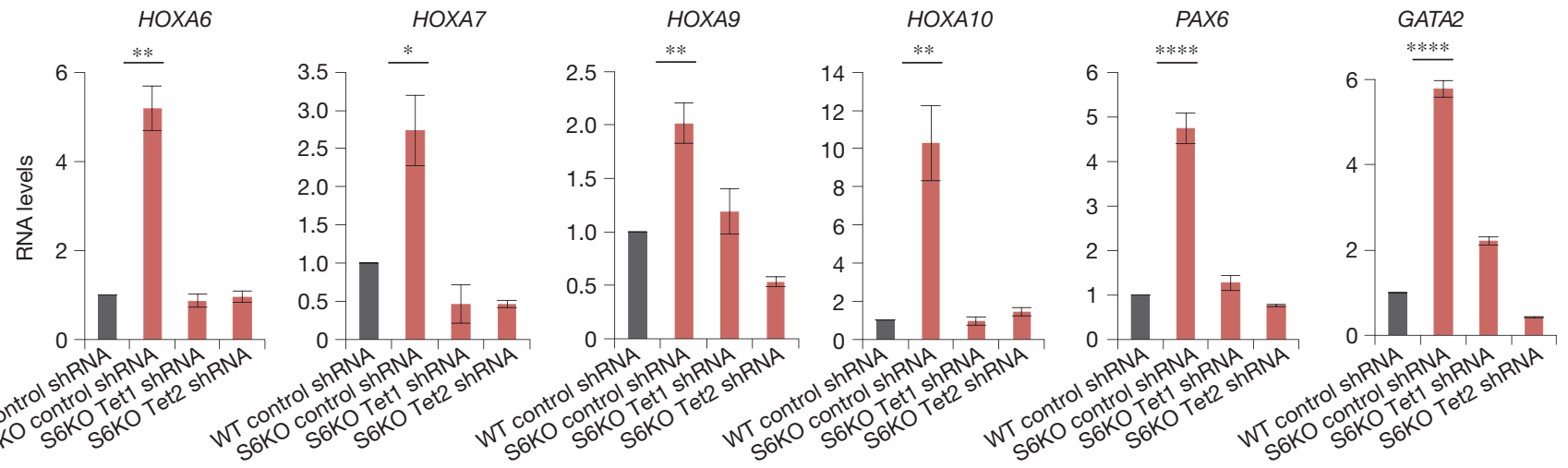

b

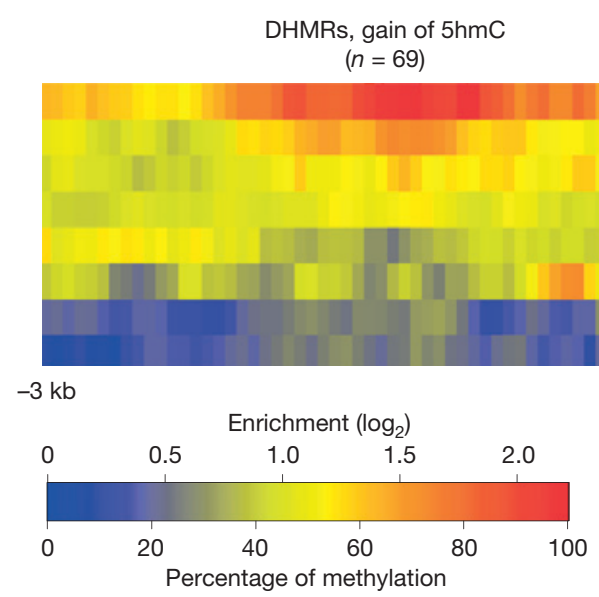

C

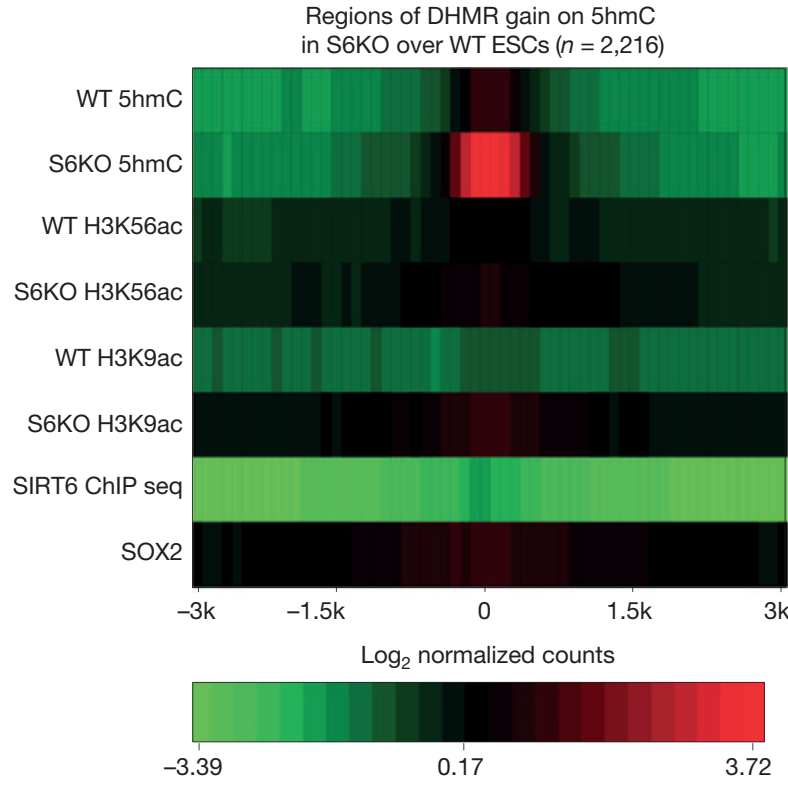

DHMRs on neuroectoderm genes

DHMRs, loss of $5 \mathrm{hmC}$ $(n=8)$

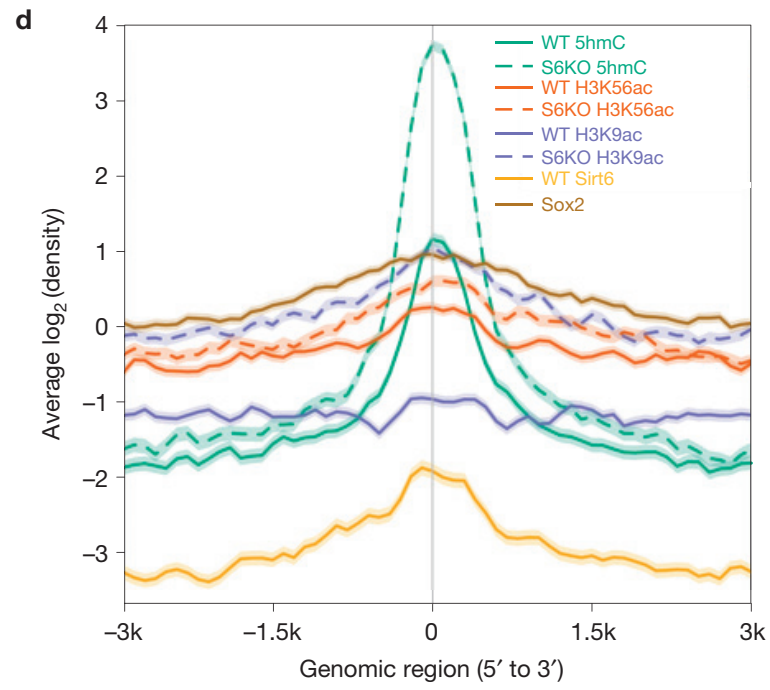

Figure 6 Association of $5 \mathrm{hmC}$ with $\mathrm{H} 3 \mathrm{~K} 4 \mathrm{me} 2$, but not $\mathrm{H} 3 \mathrm{~K} 9 \mathrm{ac}$ and/or $\mathrm{H} 3 \mathrm{~K} 56 \mathrm{ac}$, in neuroectoderm genes upregulated in S6KO compared with WT ESCs. (a) Expression of neuroectoderm genes with gain of $5 \mathrm{hmC}$ in Tet knockdown ESCs. qRT-PCR data are expressed relative to WT control shRNA. Data are $n=3$ experimental replicates (independent RNA preparations), and values are mean \pm s.e.m. ${ }^{*} P<0.05,{ }^{* *} P<0.01,{ }^{* * * *} P<0.0001$, by $t$-test analyses. (b) Enrichment analysis of histone $\mathrm{H} 3$ modifications connect $\mathrm{H} 3 \mathrm{~K} 4 \mathrm{me} 2$ to upregulated neuroectoderm genes with gain of $5 \mathrm{hmC}$ in S6KO. The marks are sorted by high (top) to low (bottom) enrichment at the centre of
DHMRs with gain of $5 \mathrm{hmC}$ in S6KO. (c) Heat map plot of regions of DHMR gain on $5 \mathrm{hmC}$ in S6KO over WT in ESCs showing average profile for $\pm 3 \mathrm{k}$ centred around the 2,216 regions with DHMR gains for the factors $5 \mathrm{hmC}$, H3K56ac, H3K9ac, SIRT6 and SOX2 in WT and S6KO ESCs. Each row of the heat map represents mean values of enrichment $z$-scores in 10-bp windows in the $\pm 3 \mathrm{~kb}$ region. (d) Enrichment line plot of average profile for regions of DHMR gain on $5 \mathrm{hmC}$ in S6KO over WT mouse ESCs $(n=2,216)$ for data in c. The semi-transparent band behind the line shows the s.e.m. for each average profile. 
Fig. 5a). By grouping gene promoters according to their $\mathrm{CpG}$ densities ( \pm 1 kilobase $(\mathrm{kb})$ around their transcription start sites), we found DHMRs to be equally enriched in low- (LCP) as well as high- (HCP) CpG-density promoters (Fig. 5a). However, gain of $5 \mathrm{hmC}$ within exons was preferentially observed at genes important for regulation of transcription and neuronal differentiation (Fig. 5b and Supplementary Table 2 and Supplementary Figs 2 and 3). Notably, the presence of $5 \mathrm{hmC}$ at exons was recently shown to positively correlate with gene expression $^{27}$. In particular, the Hoxa gene cluster, which is implicated in neural crest development, exhibits a significant enrichment of $5 \mathrm{hmC}$, along with Gata2 and Pax6, also implicated in neurogenesis (Fig. 5c). However, the housekeeping gene $\beta$-glucuronidase (Gusb) shows no difference in $5 \mathrm{hmC}$ levels between WT and S6KO ESCs (Fig. 5c), which ensures specificity in this analysis. By correlating gain or loss of $5 \mathrm{hmC}$ with publicly available data on histone marks, bisulphite-derived methylation, and transcription factor binding data (Supplementary Table 3), we found that regions that gained $5 \mathrm{hmC}$ in S6KO compared with WT ESCs were also enriched in $\mathrm{H} 3 \mathrm{~K} 4 \mathrm{me} 2$, an epigenetic mark associated with both promoters and enhancers that is involved in transcriptional activation and the binding of TET1 (Fig. 5d). Interestingly, the gain of $5 \mathrm{hmC}$ in $\mathrm{S} 6 \mathrm{KO}$, compared with WT ESCs, occurs at regions with low $5 \mathrm{mC}$ in WT ESCs (Fig. 5d), confirming an important role for SIRT6 in regulating $5 \mathrm{hmC}$ at lowmethylated regions, which have been previously associated with distal regulatory elements ${ }^{28}$ (Fig. 5d). Concomitant to the enrichment of 5hmC and $\mathrm{H} 3 \mathrm{~K} 4 \mathrm{me} 2$, we found the expression of the Hoxa gene cluster together with Gata2 and Pax6, along with other genes of the neural lineage, to be upregulated in S6KO versus WT ESCs (Fig. 6a and Supplementary Fig. 4A). Importantly, the upregulation of these genes was rescued on Tet1 or Tet2 knockdown (Fig. 6a and Supplementary Fig. 4A). The association between $5 \mathrm{hmC}$ and $\mathrm{H} 3 \mathrm{~K} 4 \mathrm{me} 2$ was further evaluated by correlation with public data as described above, on a set of genes from the neural lineage whose expression is upregulated in $\mathrm{S} 6 \mathrm{KO}$ and rescued on Tet knockdown (Fig. 6b). This analysis shows a strong association between $5 \mathrm{hmC}$ with $\mathrm{H} 3 \mathrm{~K} 4 \mathrm{me} 2$ within neuroectoderm genes whose expression is increased in S6KO versus WT ESCs (Fig. 6a,b). We also address any potential interplay of $5 \mathrm{hmC}$ with $\mathrm{H} 3 \mathrm{~K} 9 \mathrm{ac}$ and/or $\mathrm{H} 3 \mathrm{~K} 56 \mathrm{ac}$ by genome-wide analyses. Consistent with our previous work, we found a global elevation of both $\mathrm{H} 3 \mathrm{~K} 56 \mathrm{ac}$ and $\mathrm{H} 3 \mathrm{~K} 9 \mathrm{ac}$ at various genomic regions in ESCs, which is maintained after RA-dependent differentiation (Supplementary Fig. 7). We found SIRT6 and its histone targets, $\mathrm{H} 3 \mathrm{~K} 56 \mathrm{ac}$ and $\mathrm{H} 3 \mathrm{~K} 9 \mathrm{ac}$, to mark genes involved in transcription, metabolism, RNA processing, cell cycle, chromatin organization, and DNA repair as well as ESC marker genes associated with the maintenance of pluripotency (Supplementary Tables 4-8). Notably, we found no significant correlation on the enrichments of 5hmC, H3K56ac, H3K9ac, or SIRT6 binding in S6KO versus WT ESCs (Fig. 6c,d and Supplementary Fig. 8A-D). We also evaluated the role of SOX2, which was shown to promote expression of the neural lineage ${ }^{23}$, by comparing the genomic regions enriched for $5 \mathrm{hmC}$ with the SOX2 ChIP-Seq data set from ref. 29, and found no significant correlation with the gain of $5 \mathrm{hmC}$ in $\mathrm{S} 6 \mathrm{KO}$ versus WT ESCs (Fig. 6c,d). We also found no correlation of H3K56ac and H3K9ac with SOX2 targets (Supplementary Fig. 8 and Supplementary Table 9). Overall, these data indicate that enrichment of $5 \mathrm{hmC}$ in $\mathrm{S} 6 \mathrm{KO}$ is not directly interlocked with H3K56ac and/or H3K9ac, as well as SIRT6- or SOX2-targeted genomic regions. Therefore, SIRT6 plays a hierarchical role in regulating ESC differentiation by modulating TET-dependent production of $5 \mathrm{hmC}$ through direct repression of the core pluripotent gene network via deacetylation of H3K56ac and H3K9ac. Furthermore, these data strongly suggest that $5 \mathrm{hmC}$ may function as a positive transcriptional determinant to control the expression of genes associated with neuroectoderm development.

\section{SIRT6 regulates developmental programs in vivo}

To further evaluate the role of Sirt6 in ESC differentiation in vivo, we injected S6KO and WT ESCs subcutaneously into immunodeficient mice and followed teratoma formation. Teratomas derived from S6KO were significantly smaller compared with their WT counterparts (Fig. 7a). The neuroectoderm marker $\beta$-III tubulin and OCT4 were expressed at elevated levels in S6KO-derived teratomas (Fig. 7b). Remarkably, knocking down either Tet 1 or Tet 2 rescued the smaller size of teratomas derived from S6KO ESCs (Fig. 7c). Furthermore, the upregulation of OCT4, $\beta$-III tubulin and GFAP expression was also rescued on either Tet 1 or Tet 2 knockdown (Fig. $7 \mathrm{~d}, \mathrm{e})$. To explore this phenotype further, we integrated a GFP-encoding gene within the Rosa locus in both WT and S6KO iPSCs. These cells were then injected into mouse blastocysts and chimaerism was determined in mid-gestation (E12.5) embryos by immunohistochemistry (IHC) using an anti-GFP antibody. As expected, WT GFP-iPSCs gave rise to most if not all tissues of the GFP chimaeras (Fig. 8a and Supplementary Table $8 \mathrm{~B}$ ). However, mice originated from blastocysts injected with S6KO iPSCs exhibited a weak GFP staining and in some cases almost exclusively in tissues of the central nervous system, such as diencephalon and partial regions of the neural cord (marked by yellow arrows; Fig. 8a and Supplementary Table 8B).

\section{Depletion of SIRT6 in human ESCs leads to a differentiation defect similarly to mouse S6KO ESCs}

To assess whether this SIRT6-dependent developmental mechanism is evolutionarily conserved, we explored the differentiation capacity of human ESCs (hESCs) on shRNA-mediated depletion of SIRT6, and found that SIRT6-depleted human EBs (hEBs) were significantly smaller compared with their controls (Fig. 8c). Furthermore, the expression of TET1, TET2 and OCT4 was elevated in SIRT6-depleted hESCs (Fig. 8d). Paralleling the role of SIRT6 in mouse ESCs, we found from genome-wide analysis in ref. 30 that human SIRT6 is recruited at human Oct4 and Sox2 genes (Supplementary Fig. 4B), supporting an evolutionarily conserved role for SIRT6 in regulating the core pluripotent genes. Consistently, we found minimal or no binding of SIRT6 to the Tet genes (Supplementary Fig. 4C), again suggesting that SIRT6 modulates TET-dependent production of $5 \mathrm{hmC}$, indirectly. Furthermore, the neuroectoderm marker nestin was upregulated in SIRT6-deficient hESCs (Fig. 8d), supporting the predisposition towards the neural cell lineage in the absence of SIRT6. Hence, we propose a model whereby SIRT6 controls ESC differentiation by repressing the expression of Oct4 and Sox2, consequently diminishing the OCT4:SOX2-activated expression of Tet enzymes, and thereby limiting the levels of $5 \mathrm{hmC}$ at specific genomic regions to allow balanced transcription of developmentally regulated genes (Fig. 8e). 

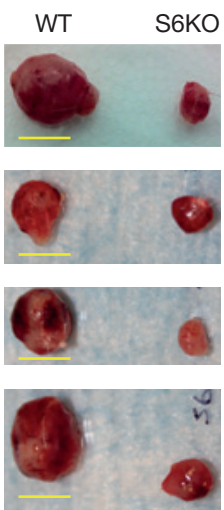

d
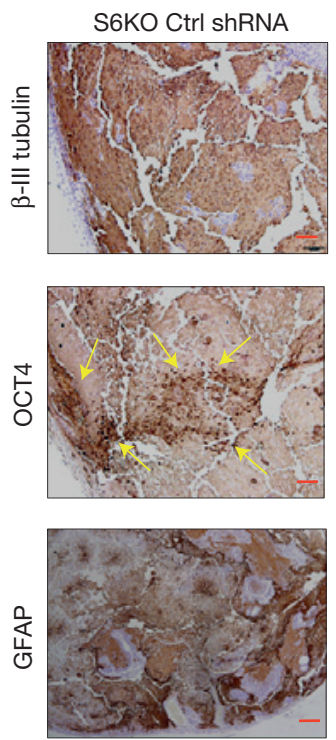
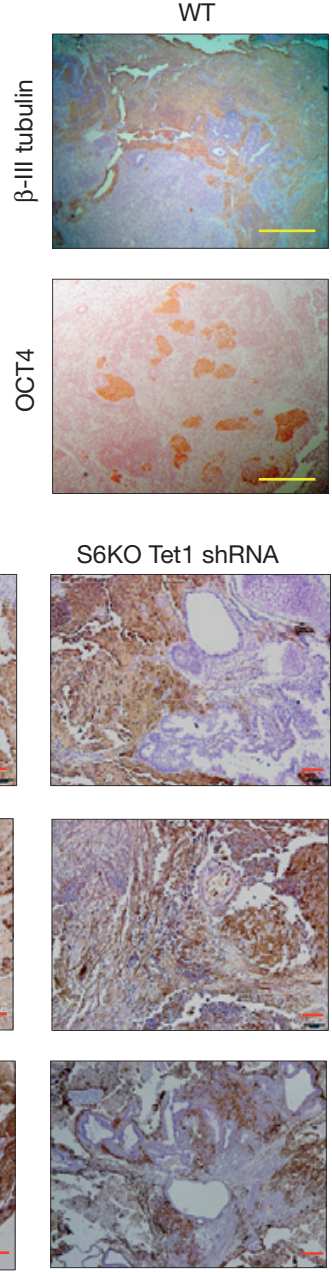
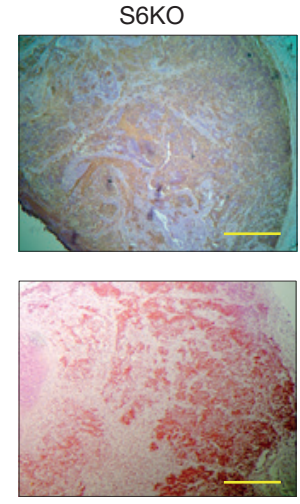

e
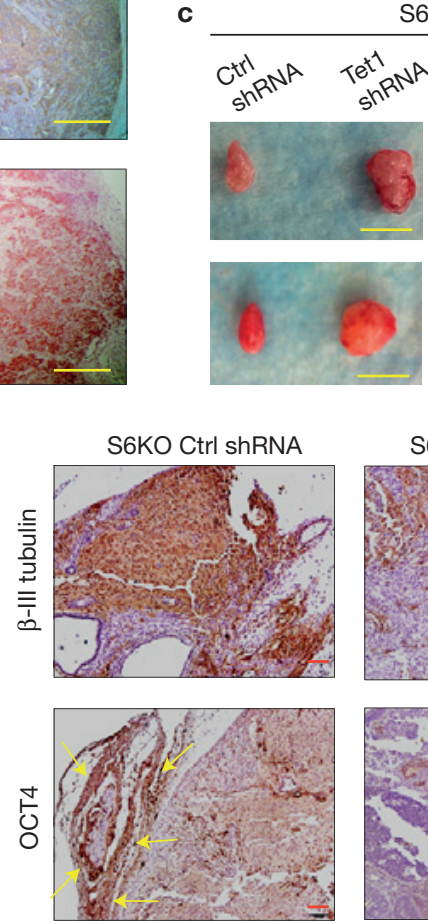

S6KO
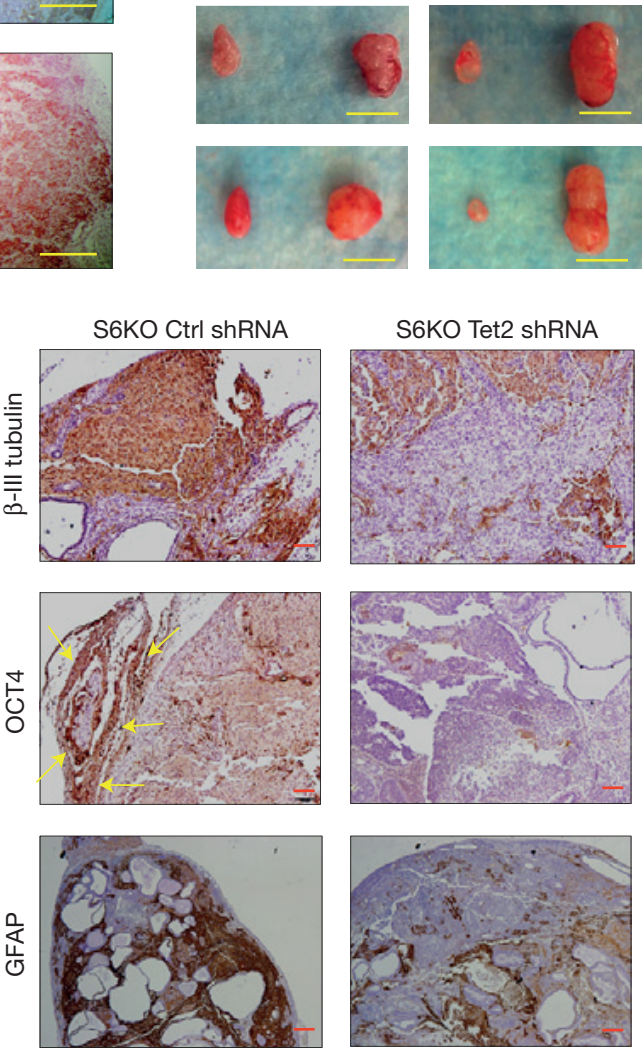

Figure 7 SIRT6 deficiency triggers an in vivo differentiation defect in mouse and in human EBs, which is rescued by Tet knockdown on mouse teratomas. (a) Teratomas from C57BL/6 WT and S6KO ESCs. Data are shown as $n=4$ biological replicates (teratomas derived from 4 distinct mice). Scale bars, $1 \mathrm{~cm}$. (b) IHC analysis for $\beta$-III tubulin and OCT4 on WT versus S6KO teratomas. Pictures are taken at $\times 5$. IHC of one representative from $n=3$ experimental replicates is

\section{DISCUSSION}

A critical step during ESC differentiation involves the silencing of the pluripotent gene network to allow expression of cell-lineagespecific genes. The core pluripotent genes Oct4 and Nanog undergo transcriptional silencing through DNA methylation at their regulatory regions, which is maintained in differentiated somatic cells ${ }^{31,32}$. Our work demonstrates an additional mechanism to repress expression of the core pluripotent genes during ESC differentiation, which involves the histone deacetylase SIRT6. Previous studies showed that SIRT6 is a critical modulator of glycolytic metabolism, DNA repair and cancer ${ }^{18-21}$. Our work determines an important function for SIRT6 as a regulator of ESC differentiation, by repressing the expression of Oct4, Sox2 and Nanog. Importantly, derepression of these core pluripotent genes in S6KO during ESC differentiation suggests that lack of SIRT6 could potentially increase the efficiency of reprogramming. Indeed, we found a $\sim 10$-fold increase in iPSC formation during reprogramming shown (teratomas derived from 3 distinct mice). Scale bars, $250 \mu \mathrm{m}$. (c) Teratomas derived from stably infected S6KO ESCs with shRNA targeting either Tet1 or Tet2 versus control shRNA. Scale bars, $1 \mathrm{~cm}$. (d,e) IHC staining for $\beta$-III tubulin, OCT4 and GFAP of teratomas from c. One representative from $n=3$ experimental replicates is shown. Areas of OCT4-positive nuclear staining are demarked by the yellow arrows. Scale bars, $100 \mu \mathrm{m}$.

of mouse neural progenitor cells derived from S6KO mice compared with WT controls (Supplementary Fig. 5F-H).

Somatic cells switch from an oxidative metabolic state to a glycolytic state during reprogramming to iPSCs (refs 33-37). Furthermore, glycolysis is critical for the maintenance of pluripotency ${ }^{38}$. Both MEFs and ESCs lacking SIRT6 exhibit a higher rate of glycolysis compared with WT (ref. 19). However, the differentiation phenotype of S6KO EBs was not rescued following glycolysis inhibition, by knocking down Pdk1 (Supplementary Fig. 5A). These data suggest a predominant role for SIRT6 in regulating the expression of pluripotent genes and $5 \mathrm{hmC}$ levels, independent of its function in metabolism, during ESC differentiation. Importantly, ectopic expression of human SIRT6 rescues the differentiation phenotype of S6KO EBs, thereby establishing specificity for the role of SIRT6 in the S6KO differentiation phenotype as well as its conserved role between mice and humans (Supplementary Fig. 6A-C). 

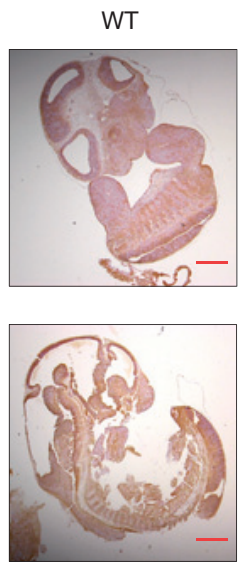

S6KO
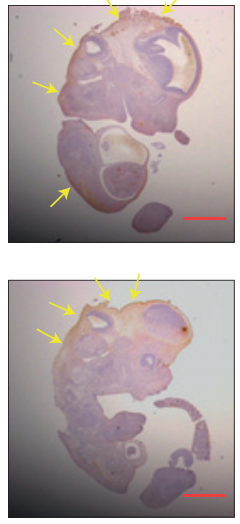

\begin{tabular}{|l|c|c|c|}
\hline \multicolumn{1}{|c|}{$\begin{array}{c}\text { ESC } \\
\text { genotype }\end{array}$} & $\begin{array}{c}\text { Embryos } \\
\text { developed }\end{array}$ & $\begin{array}{c}\text { Chimaeric } \\
\text { embryos }\end{array}$ & Degree of chimaerism \\
\hline S6KO iPSCs (1) & 21 & 6 & 3 weak, 3 neural bias \\
\hline S6KO iPSCs (2) & 16 & 7 & 2 very weak, 5 weak \\
\hline WT iPSCs & 9 & 6 & 5 strong, 1 weak \\
\hline
\end{tabular}

C
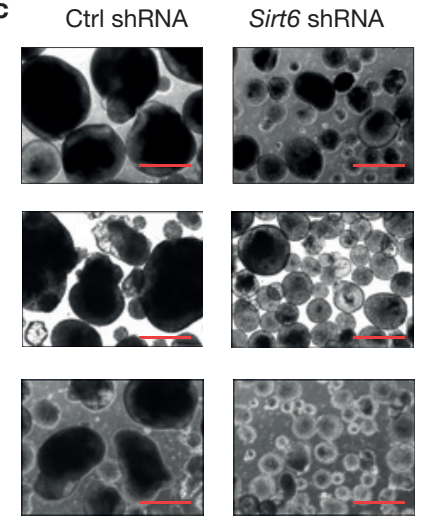

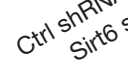

d

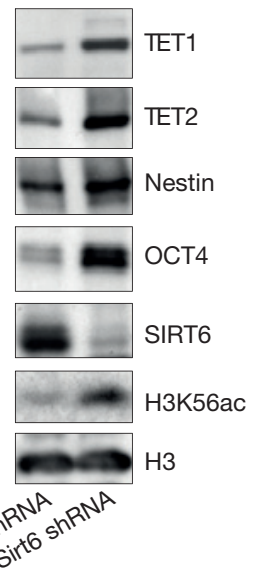

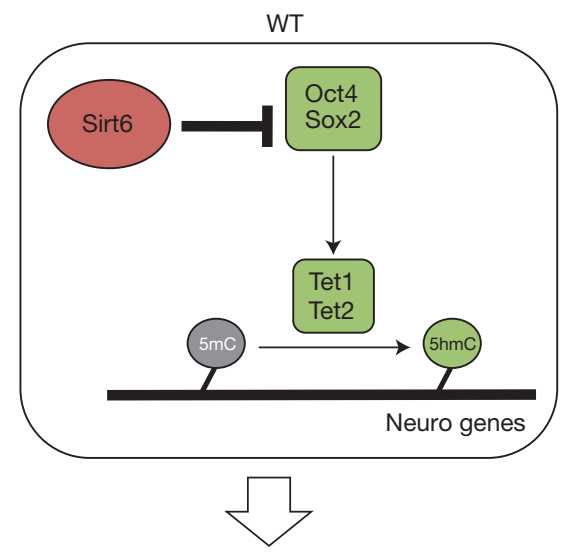

Neuroectoderm

Mesoderm

Endoderm

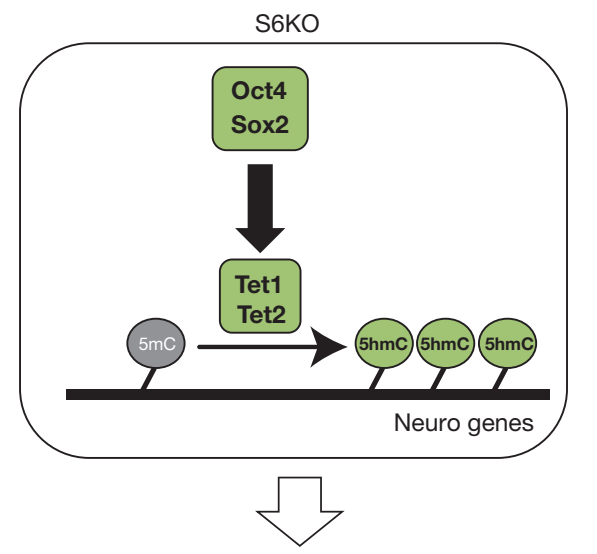

Neuroectoderm

Endoderm

Mesoderm
Figure 8 Sirt6 deficiency triggers an in vivo differentiation defect in mouse and in human EBs. (a) IHC analysis for GFP on chimaeric mice (E12.5) derived from WT or S6KO iPSCs. Note that the contribution of S6KO iPSCs is restricted to some neural tissues (yellow arrows). Scale bars, $1 \mathrm{~mm}$. (b) Table showing the numbers of embryos developed and the degree of chimaerism based on IHC with anti-GFP antibody. Note that S6KO iPSCs exhibit a weak degree of chimaerism or are restricted to the neural tissue in three embryos. (c) hEBs stably infected with an shRNA control or shRNA targeting Sirt6. Scale bars, $500 \mu \mathrm{m}$. A representative of $n=5$ biological replicates is shown. (d) Western blot analysis for Tet enzymes, Oct4 and the neuroectoderm

The biological significance of TET-mediated $5 \mathrm{mC}$ oxidation in epigenetic regulation remains poorly understood, especially with regard to its relevance during ESC differentiation, whereby pluripotent cells commit to specific cell lineages. In addition to being a DNA demethylation intermediate, $5 \mathrm{hmC}$ is recognized by chromatin regulatory proteins and therefore postulated to function as an epigenetic mark $^{39}$. However, specific target genes, whose expression is associated with $5 \mathrm{hmC}$ as an epigenetic determinant of cell lineage specification during ESC differentiation, are unknown. We identified marker nestin on hEBs stably infected with an shRNA control or shRNA targeting Sirt6. A representative from $n=3$ experimental replicates is shown. (e) Schematic representation depicting the role of Sirt6 as a regulator of ESC differentiation via repression of Oct4 and Sox2 gene expression that in turn controls Tet-dependent oxidation of $5 \mathrm{mC}$ into $5 \mathrm{hmC}$, which is needed to achieve proper development of the germ layers. Sirt6 depletion causes a derepression of Oct4 and Sox2 triggering an upregulation of Tetdependent $5 \mathrm{hmC}$ production that results in skewed development towards neuroectoderm. Uncropped images of blots are shown in Supplementary Fig. 9.

genes associated with the neural lineage whose expression is directly correlated with an enrichment of $5 \mathrm{hmC}$ at promoters and exons in S6KO ESCs (Fig. 5a-c). Furthermore, the enrichment of $5 \mathrm{hmC}$ in these genes occurs near $\mathrm{H} 3 \mathrm{~K} 4 \mathrm{me} 2$ marks, an epigenetic signature involved in transcription activation (Figs $5 \mathrm{~d}$ and $6 \mathrm{~b}$ ). The upregulated expression of TET enzymes in S6KO ESCs suggests a potential increase in further oxidized forms of $5 \mathrm{mC}$. Indeed, we detected elevated levels of $5 \mathrm{caC}$ in $\mathrm{S6KO}$ versus WT ESCs by slot blot analysis, which are rescued on Tet1 or Tet2 knockdown (Supplementary Fig. 5B-E). 
Therefore, S6KO ESCs represent a relevant biological system to further analyse the importance of TET-mediated DNA oxidation during cell fate choices.

Recent studies have shown that Tet1/Tet2 double knockout ESCs are depleted of $5 \mathrm{hmC}$, which correlates with developmental defects in teratomas and chimaeric embryos ${ }^{40}$. Tet1/Tet2-deficient mice were obtained at lower frequency indicating a phenotype of partial lethality and thereby supporting the critical role of TET enzymes in embryonic development ${ }^{40}$. In this context, S6KO mice from specific genetic backgrounds (C57BL/6) are born at lower Mendelian ratios (see Supplementary Table 11), possibly due to embryonic lethality, thereby supporting that imbalanced levels of TET proteins impair embryonic development.

Changes in overall chromatin architecture are required during the transition from pluripotency to differentiated states. However, the interplay between chromatin regulators and epigenetic determinants associated with the establishment of transcriptional programs during cell fate choices remain poorly understood. Our work identifies the chromatin enzyme SIRT6 as a key regulator of ESC differentiation, acting through sequential regulation of the core pluripotent genes and TET-mediated production of $5 \mathrm{hmC}$ to control expression of genes involved in neural cell fate. Thus, in the absence of SIRT6, neuralrelated genes are marked with elevated levels of $5 \mathrm{hmC}$, suggesting that this modification might function as either an epigenetic determinant or a facilitator of local DNA demethylation that channels ESC commitment to the neural cell lineage. Interestingly, TET2-dependent hydroxylation of $5 \mathrm{mC}$ was also found to be required for the transcriptional activation of the Hoxa cluster, which is critical for cell lineage specification in NT2 cells, an embryonic carcinoma cell line that can be differentiated with RA into neural cells ${ }^{41}$. Together, our data argue that the differentiation defect we observe in S6KO EBs is linked to Tet function, resulting in a predisposition towards a neuroectoderm developmental pathway.

Collectively, our studies unravel a molecular mechanism implicating SIRT6 as a critical regulator of ESC differentiation that involves the core pluripotent genes and TET-dependent production of $5 \mathrm{hmC}$. Future studies will determine, at a gene-by-gene level, whether $5 \mathrm{hmC}$ and its further oxidized forms $5 \mathrm{fC}$ and $5 \mathrm{caC}$ participate in gene expression and ESC differentiation as molecular intermediates in the process of DNA demethylation, as epigenetic marks that recruit chromatin and transcriptional regulators to gene regulatory regions, or whether both mechanisms apply.

\section{METHODS}

Methods and any associated references are available in the online version of the paper.

Note: Supplementary Information is available in the online version of the paper

\section{ACKNOWLEDGEMENTS}

This work was supported in part by NIH grants GM093072-01, DK088190-01A1 (R.M.), 5R01HD058013-05 (to K.H.) and HD065812, CA151535 (to A.R.). R.M. is the Kristine and Bob Higgins MGH Research Scholar, the Warshaw Institute Fellow, and a Howard Goodman Awardee. L.C. was the recipient of a Feodor Lynen Research Fellowship from the Alexander von Humboldt Foundation. Y.H. was supported by a postdoctoral fellowship from the Leukemia and Lymphoma Society. C.A.S. is the recipient of the Evans Center Fellow Award. A.Gladden and A.Goren were the recipient of the Broad Institute SPARC (Scientific Projects to Accelerate Research and Collaboration) program. We thank O. Bar-Nur and S. Cheloufi for advice on the reprogramming of neural progenitor cells, and E. Kelliher for setting up the automation of the ChIP-Seq experiments.

\section{AUTHOR CONTRIBUTIONS}

J-P.E. conceived and designed most of the experiments, collected and analysed data, and wrote the manuscript; L.C., M.L. and K.N.R. performed computational analyses; Y.H. performed ChIP-Seq experiments; J.C. performed human ESC experiments; C.A.S. and G.M. performed iPSC experiments; B.M-P. performed and analysed immunofluorescence experiments; R.M.W. performed neurogenesis and immunofluorescence experiments; A.Gladden performed ChIP-Seq experiments; S.K. performed cloning of human SIRT6 expression system; D.M.S. performed in vitro experiments; S.R. supervised computational analyses; K.H. supervised the human ESC experiments; A.Goren conceived, supervised and analysed all the ChIP-Seq experiments and their computational analysis; A.R. supervised computational analyses, $5 \mathrm{hMeC}$ Chip-Seq experiments and edited the manuscript; R.M. conceived and supervised the study, analysed the data and wrote the manuscript.

\section{COMPETING FINANCIAL INTERESTS}

The authors declare no competing financial interests.

Published online at http://dx.doi.org/10.1038/ncb3147

Reprints and permissions information is available online at www.nature.com/reprints

1. Chen, T. \& Dent, S. Y. Chromatin modifiers and remodellers: regulators of cellular differentiation. Nat. Rev. Genet. 15, 93-106 (2014).

2. Smith, Z. D. \& Meissner, A. DNA methylation: roles in mammalian development. Nat. Rev. Genet. 14, 204-220 (2013)

3. Iyer, L. M., Tahiliani, M., Rao, A. \& Aravind, L. Prediction of novel family of enzymes involved in oxidative and other complex modifications of bases in nucleic acids. Cell Cycle 11, 1698-1710 (2009).

4. Tahiliani, M. et al. Conversion of 5-methylcytosine to 5-hydroxymethylcytosine in mammalian DNA by MLL partner TET1. Science 324, 930-935 (2009).

5. He, Y. F. et al. Tet-mediated formation of 5-carboxylcytosine and its excision by TDG in mammalian DNA. Science 333, 1303-1306 (2011).

6. Ito, S. et al. Tet proteins can convert 5-methycytosine to 5-formylcytosine and 5carboxylcytosine. Science 333, 1300-1303 (2011).

7. Ficz, G. et al. Dynamic regulation of 5-hydroxymethylcytosine in mouse ES cells and during differentiation. Nature 473, 398-402 (2011).

8. Williams, K. et al. TET1 and hydroxymethylcytosine in transcription and DNA methylation fidelity. Nature 473, 343-348 (2011).

9. Wu, H. et al. Genome-wide analysis of 5-hydroxymethylcytosine distribution reveals its dual function in transcriptional regulation in mouse embryonic stem cells. Genes Dev. 25, 679-684 (2011).

10. Kriaucionis, S. \& Heintz, N. The nuclear DNA base 5-hydroxymethylcytosine is present in Purkinje neurons and the brain. Science 324, 929-930 (2009).

11. Ito, S. et al. Role of Tet proteins in $5 \mathrm{mC}$ to $5 \mathrm{hmC}$ conversion, ES-cell self-renewal and inner cell mass specification. Nature 466, 1129-1133 (2010).

12. Ko, M. et al. Impaired hydroxylation of 5-methylcytosine in myeloid cancers with mutant TET2. Nature 468, 839-843 (2010).

13. Szwagierczak, A., Bultmann, S., Schmidt, C. S., Spada, F. \& Leonhardt, H. Sensitive enzymatic quantification of 5-hydroxymethylcytosine in genomic DNA. Nucleic Acids Res. 38, e181 (2010).

14. Koh, K. P. et al. Tet1 and tet2 regulate 5-hydroxymethylcytosine production and cell lineage specification in mouse embryonic stem cells. Cell Stem Cell $\mathbf{8}$, 200-213 (2011).

15. Xie, W. et al. Histone $\mathrm{H} 3$ lysine 56 acetylation is linked to the core transcriptional network in human embryonic stem cells. Mol. Cell 33, 417-427 (2009).

16. Yang, B., Zwaans, B. M. M., Eckersdoroff, M. \& Lombard, D. B. The sirtuin Sirt6 deacetylates H3K56Ac in vivo to promote genomic stability. Cell Cycle 16, 2662-2663 (2009).

17. Michishita, E. et al. Cell cycle-dependent deacetylation of telomeric histone H3 lysine K56 by human Sirt6. Cell Cycle 16, 2664-2666 (2009).

18. Mostoslavsky, R. et al. Genomic instability and aging-like phenotype in the absence of mammalian SIRT6. Cell 124, 315-329 (2006).

19. Zhong, L. et al. The histone deacetylase SIRT6 regulates glucose homeostasis via Hif1a. Cell 140, 280-293 (2010).

20. Sebastián, C. et al. The histone deacetylase SIRT6 is a tumor suppressor that controls cancer metabolism. Cell 151, 1185-1199 (2012).

21. Toiber, D. et al. SIRT6 recruits SNF2H to DNA break sites, preventing instability through chromatin remodeling. Mol. Cell 51, 454-468 (2013).

22. Young, R. A. Control of the embryonic stem cell state. Cell 144, 940-954 (2011).

23. Thomson, M. et al. Pluripotency factors in embryonic stem cells regulate differentiation into germ layers. Cell 145, 875-889 (2011).

24. Wu, Y. et al. Oct4 and the small molecule inhibitor, SC1, regulates Tet2 expression in mouse embryonic stem cells. Mol. Biol. Rep. 40, 2897-2906 (2013).

25. Pastor, W. A. et al. Genome-wide mapping of 5-hydroximethylcytosine in embryonic stem cells. Nature 473, 394-397 (2011). 
26. Lienhard, M., Grimm, C., Morkel, M., Herwig, R. \& Chavez, L. MEDIPS: genomewide differential coverage analysis of sequencing data derived from DNA enrichment experiments. Bioinformatics 30, 284-286 (2014).

27. Huang, Y. et al. Distinct roles of the methylcytosine oxidases Tet1 and Tet2 in mouse embryonic stem cells. Proc. Natl Acad. Sci. USA 111, 1361-1366 (2014).

28. Stadler, M. B. et al. DNA-binding factors shape the mouse methylome at distal regulatory regions. Nature 480, 490-495 (2011).

29. Lodato, M. A. et al. SOX2 co-occupies distal enhancer elements with distinct POU factors in ESCs and NPCs to specific cell state. PLoS Genet. 9, e1003288 (2013)

30. Ram, O. et al. Combinatorial patterning of chromatin regulators uncovered by genome-wide location analysis in human cells. Cell 147, 1628-1639 (2011).

31. Epsztejn-Litman, S. et al. De novo DNA methylation promoted by G9a prevents reprogramming of embryonically silenced genes. Nat. Struct. Mol. Biol. 15, 1176-1183 (2008)

32. Li, J. Y. et al. Synergistic function of DNA methyltransferases Dnmt3a and Dnmt3b in the methylation of Oct4 and Nanog. Mol. Cell. Biol. 27, 8748-8759 (2007)

33. Folmes, C. D. et al. Somatic oxidative bioenergetics transitions into pluripotencydependent glycolysis to facilitate nuclear reprogramming. Cell Metab. 14, 264-271 (2011).
34. Panopoulos, A. D. et al. The metabolome of induced pluripotent stem cells reveals metabolic changes occurring in somatic cell reprogramming. Cell Res. 22, 168-177 (2012).

35. Prigione, A. \& Adjaye, J. Modulation of mitochondrial biogenesis and bioenergetic metabolism upon in vitro and in vivo differentiation of human ES and iPS cells. Int. J. Dev. Biol. 54, 1729-1741 (2010).

36. Varum, S. et al. Energy metabolism in human pluripotent stem cells and their differentiated counterparts. PLOS ONE 6, e20914 (2011).

37. Zhou, W. et al. HIFla induced switch from bivalent to exclusively glycolytic metabolism during ESC-to-EpiSC/hESC transitions. EMBO J. 31, 2103-2116 (2012).

38. Zhang, J., Nuebel, E., Daley, G. Q., Koehler, C. M. \& Teitell, M. A. Metabolic regulation in pluripotent stem cells during reprogramming and self-renewal. Cell Stem Cell 11, 589-595 (2012).

39. Spruijt, C. G. et al. Dynamic readers for 5-(hydroxy)methylcytosine and its oxidized derivatives. Cell 152, 1146-1159 (2013)

40. Dawlaty, M. M. et al. Combined deficiency of Tet1 and Tet2 causes epigenetic abnormalities but is compatible with postnatal development. Cell 24, 310-323 (2013).

41. Bocker, M. T. et al. Hydroxylation of 5-methylcytosine by TET2 maintains the active state of the mammalian HOXA cluster. Nat. Commun. 3, 818 (2012). 


\section{METHODS}

Mouse ESC cultures, formation and immunostaining of embryoid bodies. Mouse ESCs (mESCs) derived from Sirt6 KO and WT 129 mouse strain ${ }^{18}$ were maintained on $\gamma$-irradiated mouse embryonic fibroblasts (MEFs) in knockout DMEM medium (GIBCO) containing 15\% ES-qualified FBS, $0.1 \mathrm{mM}$ each of nonessential amino acids, $2 \mathrm{mM}$ L-glutamine, $0.1 \mathrm{mM} \beta$-mercaptoethanol, 50 units $\mathrm{ml}^{-1}$ penicillin/streptomycin (Invitrogen) and supplemented with leukaemia inhibiting factor (LIF). For all experiments described, cells were trypsinized and plated for $30 \mathrm{~min}$ on standard tissue culture dishes to remove feeder cells before floating ESCs were collected and re-plated on gelatin-coated dishes or wells before differentiation towards embryoid bodies (EBs). ESCs were dissociated with trypsin (day 0) and cultured at a density of 5,000 cells ml ${ }^{-1}$ in Iscove's modified Dulbecco's medium (IMDM) with 15\% FCS (Atlanta Biologicals), 10\% protein-free hybridoma medium (PFHM-II; Gibco), $2 \mathrm{mM} \mathrm{L-glutamine} \mathrm{(Gibco),} 200 \mu \mathrm{g} \mathrm{ml}^{-1}$ transferrin (Roche), $0.5 \mathrm{mM}$ ascorbic acid (Sigma) and $4.5^{\circ}-10^{-4} \mathrm{M}$ monothioglycerol (MTG; Sigma). Differentiation was carried out using the standard hanging drop method and EBs were collected at day 10. For immunofluorescence analysis, EBs were fixed and cryopreserved according to the procedure described in ref. 42. Gfap immunofluorescence on EBs was performed according to the procedure described in ref. 43. Image acquisition was done in a Leica fluorescence microscope under non-saturating exposure conditions. The quantification of Gfap intensity per EB was performed with CellProfiler software. Briefly, images were segmented using DAPI staining to generate masks matching EBs. This mask was applied in the Gfap image to calculate the mean intensity of Gfap staining in each EB.

Generation of Tet1 and Tet2 knockdown ESC lines. Tet1 and Tet2 shRNA constructs were used as previously described ${ }^{14}$. Briefly, ESCs were transfected by electroporation at $320 \mathrm{~V}, 250 \mu \mathrm{F}$ with Tet1, Tet2 or control pSuper.puro shRNA constructs and selected on puromycin-resistant $\gamma$-irradiated feeders. Clones were picked and screened by qRT-PCR for knockdown of Tet1 and Tet2 expression. Established stable clones were subsequently maintained in the absence of puromycin without loss of knockdown assessed after up to 10 serial passages.

Hydroxymethylated DNA immunoprecipitation assay. Short reads derived from S6KO and WT ESC cytosine 5-methylenesulphonate immunoprecipitation (CMS IP) and input assays were mapped against $\mathrm{mm} 9$ as previously described ${ }^{25}$. After discarding all reads that map to multiple positions in the genome, there are $11,679,660$ reads for S6KO CMS IP, 13,916,523 reads for WT CMS IP, 24,157,471 reads for S6KO input, and 16,743,260 reads for WT input.

To identify genome-wide differentially hydroxymethylated regions (DHMRs) in S6KO compared with WT, we calculated differential coverage at genomewide 500-bp windows by employing the Bioconductor package MEDIPS (ref. 26) v1.12.0 (extend $=200$, uniq $=\mathrm{T}$, window_size $=500$, BSgenome $=$ BSgenome.Mmusculus.UCSC.mm9, adj $=$ F, diff.method $=$ edgeR, $P$ value $=1 \times$ $10^{-3}$ ), resulting in 1,562 genomic windows with significant loss of $5 \mathrm{hmC}$ in $\mathrm{S} 6 \mathrm{KO}$, and 2,218 genomic windows with significant gain of $5 \mathrm{hmC}$ (Supplementary Table 1).

To assign DHMRs to genes, we downloaded the $\mathrm{mm} 9$ table refGene (track RefSeqGenes) via the Table Browser at the UCSC Genome Bioinformatics Site (20 December 2013). To assign DHMRs to CpG islands, we downloaded the $\mathrm{mm} 9$ table cpgIslandExt (track CpG Island) via the Table Browser at the UCSC Genome Bioinformatics Site (20 December 2013). To assign DHMRs to enhancers, we downloaded $\mathrm{mm} 9$ enhancers provided at http://chromosome.sdsc.edu/mouse/ download/mESC.zip (ref. 44). We assigned DHMRs to promoters if they overlap with the $\pm 1 \mathrm{~kb}$ region around a transcription start site. We have calculated the CpG observed/expected ratio for all gene promoters by considering the number of Cs, Gs and CpGs in the $\pm 1 \mathrm{~kb}$ region around their transcription start sites. By fitting a Gamma Normal Mixture to the resulting promoter CpG observed/expected ratios, we have identified the intersection of the Gamma and the Gaussian distributions at $\mathrm{CpG}$ obs/exp $=0.399$ as a threshold to distinguish gene promoters into lowCpG-density and high-CpG-density promoters. We assigned DHMRs to exons, CpG islands or enhancers, respectively, if their genomic coordinates overlap. Enrichment of GO terms associated with the previously identified genes containing DHMRs in their promoters or exons has been calculated using DAVID (ref. 45; Supplementary Table 2).

We tested the enrichment of DNA methylation, transcription factor binding sites, and several histone modifications within and surrounding our previously defined DHMRs (Figs $4 \mathrm{~d}$ and $5 \mathrm{c}$ ). For this, we accessed publicly available data sets as listed in Supplementary Table 3. External data sets were downloaded from NCBI GEO/SRA as sra-lite packages according to the given access numbers. Fastq files were extracted using the sra toolkit ${ }^{46}$ sratoolkit.2.1.9-centos_linux64/fastq-dump). Fastq files of the individual lanes were mapped against $\mathrm{mm} 9$ using bowtie ${ }^{47}$ ( bowtie-0.12.7/bowtie-q$n$ 2-k 1-best-strata-m 1-maxbts 10000 mm9-y-chunkmbs 1028-sam). For each data set, the mapping results of the individual lanes were sorted, pooled, and exported as one bam file using samtools ${ }^{48}$ (SAMtools-0.1.18/samtools).
Alignments (bam files) and our previously defined DHMRs (annotations) were imported into the R/Bioconductor environment (http://www.r-project.org, http: //www.bioconductor.org). Alignments were shifted 60 bases towards their $3^{\prime}$-end to account for an estimated fragment length of 120 base pairs. For each tested annotation and for each alignment, coverage was calculated as follows: the midpoint of each region associated with an annotation was calculated and the range of $-3 \mathrm{~kb}$ to $+3 \mathrm{~kb}$ around this midpoint was divided into 60 bins of constant length (bins have a length of $50 \mathrm{bp}$ ). Alignment coverage was calculated at these bins. To avoid division by zero, we added one to each bin of the immunoprecipitate and of the control sample. Subsequently, immunoprecipitate signals at the tested bins were divided by a library size correction value (see below). Enrichment is defined by the $\log _{2}$ of corrected immunoprecipitate signals divided by the control signals, where the IP data sets in Supplementary Table 3 are considered as immunoprecipitate and two separate ChIP-Seq input data sets are considered as control as indicated. For the average enrichment profiles, we calculated the $\log _{2}$ of the mean over all corrected immunoprecipitate over control ratios at every tested position of stacked annotations. For each immunoprecipitate sample, the correction value for the library size was estimated by calculating the mean over the ratios of immunoprecipitate over control sample short-read coverage at genome-wide 100-bp windows.

For DNA methylation data, we have accessed the processed single CpG table given in ref. 28 and for each tested bin we calculated the mean $\mathrm{mCpG}$ signal (that is, the mean of nMeth/nTot* 100 values of all CpGs that fall into the bin). For the ChIP-chip data set ${ }^{28}$ we accessed the available processed $\log _{2}$ ratios, calculated the mean for each probe over both replicates, and for each tested bin, we calculated the mean of included probes.

Sirt6 ChIP-Seq data analysis. To establish the most suitable conditions for Sirt6 ChIP-Seq analysis, we first tested four different antibodies by western blotting (Aviva System Biology, OAAB16579; Abcam, ab62739; Cell Signaling Technology, 12486; Sigma-Aldrich, S4322). Next, as western blot and ChIP-Seq conditions may differ, we systematically determined the antibody and concentrations that produce the highest signal-to-noise ratio. To reduce experimental variability, we used our automated ChIP-Seq methodology ${ }^{49}$. For each of the two most promising antibodies-Aviva System Biology, OAAB16579 and Cell Signaling Technology, 12486-we split the sheared chromatin between 3 ratios of antibody/chromatin $(0.5 \mathrm{ml}, 1 \mathrm{ml}$ and $5 \mathrm{ml}$ of each antibody $/ 3 \times 10^{6}$ cells), and performed ChIP-Seq. In addition, as a positive control, we used the same chromatin preparation for ChIP-Seq with an antibody targeting H3K27ac (Cell Signaling Technologies D5E4, optimized at $1 \mathrm{ml} / 20 \times 10^{6}$ cells). Our results indicated that CST in a ratio of $1 \mathrm{ml} / 20 \times 10^{6}$ cells outperformed the rest, and we used the data for further analysis.

Reads from Sirt6 ChIP-Seq for Sirt6 wild-type (S6WT) and knockout (S6KO) and WCE control for mouse ES cells were aligned to mouse genome $\mathrm{mm} 9$ using bwa and duplicate reads were marked with Picard tools (http://picard.sourceforge.net). Peaks were called using MACS2 (ref. 50) with the false discovery rate $q=0.01$. Called peaks were checked visually with the IGV browser ${ }^{51}$. Peaks were assigned to genes using the TSS and NDG methods of PeakAnnotator in the PeakAnalyzer package where genes with peaks in the promoters were defined to be those genes with a peak within a distance of $\pm 1 \mathrm{~kb}$ from the TSS and genes with a peak in an exon were defined as genes with the 'Overlap-Center' falling in the gene's exon.

Sox2 ChIP-Seq analysis. Data for Sox2 ChIP-Seq from mouse ESCs was downloaded from GEO (GSM1050291 or SRR630006.sra for Sox2 ChIP-Seq in mESC, GSM1050292 or SRR630007.sra for mESC WCE rep1, and GSM1050293 or SRR630008.sra for mESC WCE rep2; ref. 29) in the form of SRA files. SRA files were converted to fastq files using fastq-dump. Trimming of low-quality reads and clipping of sequencing adaptors was done using the program Trimmomatic ${ }^{52}$. Trimmed reads from Sox 2 ChIP-Seq in mouse ESCs and WCE control were aligned to mouse genome mm9 using bowtie ${ }^{47}$ (bowtie -q -p 11-phred33-quals -v 2 -e 70 -m 3 -best-strata-sam) and samtools ${ }^{48}$ was used to remove duplicate reads, sort and index bam files, and merge the two WCE controls. Peaks were called using MACS2 (ref. 50) with the false discovery rate $q=0.01$. Called peaks were checked visually with the IGV browser ${ }^{51}$. Peaks were assigned to genes using the TSS and NDG methods of PeakAnnotator in the PeakAnalyzer package where genes with peaks in the promoters were defined to be those genes with a peak within a distance of $\pm 1 \mathrm{~kb}$ from the TSS and genes with a peak in an exon were defined as genes with the 'Overlap-Center' falling in the gene's exon.

H3K56ac and H3K9ac ChIP-Seq data analysis. Reads from H3K56ac and H3K9ac ChIP-Seq for Sirt6 wild-type (S6WT) and knockout (S6KO) and WCE control for mouse ESCs (both untreated and treated with differentiating agent retinoic acid (RA)) were aligned to mouse genome $\mathrm{mm} 9$ using bwa and duplicate reads were marked with Picard tools (http://picard.sourceforge.net). R Bioconductor tool MEDIPS was used to find differential ChIP-Seq binding profiles for the histone marks between S6WT and S6KO in both untreated and RA-treated 
samples (using MEDIPS parameters: extend $=200$, uniq $=\mathrm{T}$, window_size $=500$, BSgenome = BSgenome.Mmusculus.UCSC.mm9, adj = F, diff.method = edgeR, $P$ value $\left.=1 \times 10^{-3}\right)$. Adjacent blocks of gain or loss were merged using the MEDIPS function mergeFrames. This resulted in genomic windows with significant loss and gain of histone marks H3K56ac and H3K9ac as shown in Table 1. as follows:

Table 1 Genomic windows with gain and loss of H3K56ac and H3K9ac.

\begin{tabular}{lll} 
& $\begin{array}{l}\text { Number of merged S6KO } \\
\text { gain blocks }\end{array}$ & $\begin{array}{l}\text { Number of merged S6KO } \\
\text { loss blocks }\end{array}$ \\
\hline H3K56ac & 18,722 & 22,126 \\
H3K9ac & 39,096 & 28,450 \\
H3K56ac with RA & 14,887 & 11,536 \\
H3K9ac with RA & 23,794 & 24,695 \\
\hline
\end{tabular}

Differential H3K56ac and H3K9ac blocks were checked visually with the IGV browser ${ }^{51}$. The merged blocks of differential gain or loss from MEDIPS were assigned to genes using the TSS and NDG methods of PeakAnnotator in the PeakAnalyzer package where genes with blocks with gains or losses in the promoters were defined to be those genes where a merged differential block fell within a distance of $\pm 1 \mathrm{~kb}$ from the TSS and genes with gains or losses in an exon were defined as genes where the 'Overlap-Center' fell in the gene's exon.

Summary ChIP-Seq heat map images. Average binding profiles for areas of DHMR gain on $5 \mathrm{hmC}$ in S6KO (Supplementary Fig. 12A,B) and histone mark gain (Supplementary Fig. 12C-F) were produced by calculating the mean coverage values of 100-bp windows in the $\pm 3 \mathrm{~kb}$ region centred in each of the areas of S6KO gain. The heat maps and line plots represent the mean enrichment by bin across all areas of S6KO gain after correcting for the direction of the DNA strand. The semi-transparent bands behind the lines of Supplementary Fig. 12B,D,F represent the standard error of the mean across the $\pm 3 \mathrm{~kb}$ region.

Quantification of Nanog in mESCs. ESCs were grown in 96-well clear-bottom plates (BD Falcon) that were pretreated with $0.2 \%$ gelatin. Cells were fixed in PBS $4 \%(\mathrm{w} / \mathrm{v})$ paraformaldehyde. Next, cells were permeabilized with PBS $0.5 \%(\mathrm{v} / \mathrm{v})$ Triton X-100, washed with PBS $0.2 \%(\mathrm{v} / \mathrm{v})$ Tween 20 and blocked with PBS $0.2 \%$ $(\mathrm{v} / \mathrm{v})$ Tween $20,5 \%$ goat serum. Cells were incubated with a 1:100 dilution of antiNanog antibody (Abcam) for $1 \mathrm{~h}$ at room temperature in blocking solution. Cells were washed 3 times with PBS $0.2 \%(\mathrm{v} / \mathrm{v})$ Tween 20 and subsequently incubated with secondary antibody conjugated with Alexa 488 for $1 \mathrm{~h}$ at room temperature in blocking solution in the dark. After 2 washes, cell were stained with $300 \mathrm{nM}$ DAPI for $2 \mathrm{~min}$, washed once with PBS and stored in PBS at $4^{\circ} \mathrm{C}$ until image acquisition. For quantification of Nanog intensity in ESCs, 16 pictures of each well were automatically acquired in a BD-Pathway 435 high-throughput microscope (Becton Dickinson) with AttoVision 1.5 software using a dry $\times 40$ magnification lens. Images were analysed with CellProfiler software. Briefly, images were segmented using the DAPI staining to generate masks matching cell nuclei. This mask was applied in the Nanog image to calculate the mean intensity of Nanog staining in each ESC. All of the images for quantitative analyses were acquired under non-saturating exposure conditions.

Human embryonic stem cell culture. Human ESC lines were cultured with MEFs (Globalstem) pre-plated at $12-15,000$ cells cm$^{-2}$. Medium containing DMEM/F12, $20 \%$ knockout serum replacement, $1 \mathrm{mM}$ L-glutamine, $100 \mu \mathrm{M}$ MEM non-essential amino acids, and $0.1 \mathrm{mM} \beta$-mercaptoethanol was used. FGF-2 at $10 \mathrm{ng} \mathrm{ml}^{-1}$ was added after sterile filtration and cells were fed daily and passaged every week using StemPro EZPassage (Invitrogen).

Human EB formation. Human ESC lines were mechanically dissociated using StemPro EZPassage (Invitrogen) to make clumps of consistent size. Human EBs were generated in suspension culture over a low-speed shaker for 10 days. Culturing conditions included medium containing DMEM/F12, 20\% knockout serum replacement, $1 \mathrm{mM}$ L-glutamine, $100 \mu \mathrm{M}$ M MEM non-essential amino acids and $0.1 \mathrm{mM} \beta$-mercaptoethanol, in the absence of FGF-2.

Generation of ESCs from C57BL/6. Four- to five-week-old Sirt6 \pm female mice (C57BL/6) were induced to superovulation (7.5 I.U. PMSG administration followed, $48 \mathrm{~h}$ after, by 7.5 I.U. hCG administration via intraperitoneal injection) and inbred. Blastocysts were collected at day 3.5 after vaginal plug check, and then cultured in knockout DMEM medium (GIBCO) as ESCs. Briefly, pre-implanted E3.5 blastocysts were retrieved from the uterine horn by flushing and collected under a dissection microscope. Each blastocyst was plated onto MEF feeder (Globalstem) containing NDiff 227 medium (StemCells) supplemented with LIF and two inhibitors ${ }^{53}$. The cells were cultured for 4 days without changing medium. After that, the cells were cultured for an additional 3-4 days and fed daily with fresh medium until picking. Outgrowth from the culture was picked, trypsinized, and re-plated onto MEF feeders containing KO DMEM (Invitrogen) supplemented with 15\% FBS (Hyclone), $1 \mathrm{mM}$ L-glutamine, $100 \mu \mathrm{M}$ M MEM non-essential amino acids, and $0.1 \mathrm{mM} \beta$-mercaptoethanol, and LIF.

Generation of iPSCs. MEFs were collected from E13.5 WT and S6KO embryos using standard procedures. iPSC lines were generated with the STEMCCA lentiviral reprogramming system essentially as described previously ${ }^{54}$. Briefly, MEFs were coinfected at passage three or four with the Dox-inducible pHAGE2-Tet-STEMCCA vector and a lentivirus expressing the reverse tetracycline transcriptional activator (rtTA) and cultured on mitomycin C-treated fibroblasts in ESC medium (DMEM supplemented with 15\% FBS, L-glutamine, penicillin/streptomycin, non-essential amino acids, $\beta$-mercaptoethanol and $1,000 \mathrm{U} \mathrm{ml}^{-1}$ LIF (ESGRO; Chemicon; Millipore). Doxycycline was added at a final concentration of $1 \mu \mathrm{g} \mathrm{ml}^{-1}$ and removed at day 10 post-infection. iPSC colonies were mechanically isolated five days following Dox removal, expanded in ESC medium and characterized by immunofluorescence and alkaline phosphatase staining.

Neurogenesis assay. ESCs were dissociated using trypsin (0.05\%) and purified by attachment to tissue culture dishes for $1 \mathrm{~h}$. EBs were allowed 3 to 4 days to form after plating of ESCs in bacterial dishes in DMEM media containing 10\% defined FBS (Sigma-Aldrich), $2 \mathrm{mM}$ L-glutamine (Invitrogen), $1 \times$ NEAA (Invitrogen), $10 \mathrm{mM}$ Hepes (Invitrogen), $1 \mathrm{mM}$ 2-mercaptoethanol, $100 \mathrm{units} \mathrm{m}^{-1}$ penicillin, and $100 \mathrm{~g} \mathrm{ml}^{-1}$ streptomycin (Invitrogen) (EB media). EBs were allowed one day to attach to tissue culture dishes, and neuronal precursors were then selected for by incubation in DMEM/F-12 media containing apotransferrin $\left(50 \mathrm{~g} \mathrm{ml}^{-1}\right.$; Sigma-Aldrich), insulin $\left(5 \mathrm{~g} \mathrm{ml}^{-1}\right.$; Sigma-Aldrich), sodium selenite ( $30 \mathrm{nM}$; Sigma-Aldrich), fibronectin ( $250 \mathrm{ng} \mathrm{ml}^{-1}$; Sigma-Aldrich), 100 units $^{-1}$ penicillin, and $100 \mathrm{~g} \mathrm{ml}^{-1}$ streptomycin (Invitrogen) (ITSFn media) for 7-10 days. Cells were subsequently dissociated by trypsin (0.05\%), and neuronal precursors were expanded and patterned for 4 days after plating onto fibronectincoated/polyornithine-coated plates at a density of 75,000 cells per square centimetre in DMEM/F-12 media containing apotransferrin $\left(100 \mathrm{~g} \mathrm{ml}^{-1}\right)$, insulin $\left(5 \mathrm{~g} \mathrm{ml}^{-1}\right)$, sodium selenite $(30 \mathrm{nM})$, progesterone $(20 \mathrm{nM})$, putrescine $(100 \mathrm{nM})$, penicillin (100 units $\left.\mathrm{ml}^{-1}\right)$, streptomycin $\left(100 \mathrm{~g} \mathrm{ml}^{-1}\right)$, laminin $\left(1 \mathrm{~g} \mathrm{ml}^{-1}\right)$, basic fibroblast growth factor (FGF2; $10 \mathrm{ng} \mathrm{ml}^{-1}$ ) (R \& D Systems), Shh (500 $\left.\mathrm{ng} \mathrm{m}^{-1}\right)(\mathrm{R} \& \mathrm{D}$ Systems), and FGF8 (100 $\mathrm{ng} \mathrm{m}^{-1}$ ) (R \& D Systems) (N3 media). The cells were subsequently differentiated in N3 media containing $200 \mathrm{M}$ ascorbic acid for 314 days (stage 5). Cells used for immunofluorescent staining were fixed in $4 \%$ formaldehyde for $20 \mathrm{~min}$ and rinsed three times with PBS followed by an overnight incubation at $4{ }^{\circ} \mathrm{C}$ with anti-TUJ1 ( $\beta$-III tubulin) antibody. Samples were then incubated with Alexa Fluor 590 secondary antibody for $2 \mathrm{~h}$ and images were captured on a Leica Microscope.

Western blot analysis. Western analysis was carried out as previously described ${ }^{19}$. The antibodies used are as follows: anti-Sirt6 (Abcam, ab62739), anti-Oct4 (Santa Cruz, sc5279), anti-Sox2 (Millipore, AB5603), anti-Nanog (Abcam, ab80892), anti-Tet1 (Millipore, 09-872), anti-Tet2 (Abcam, ab94580), anti-nestin (Abcam, ab6142), anti-H3K56ac (Abcam, abab76307), anti-H3 (Abcam, ab1791) and antiactin (Sigma-Aldrich, A2066).

Slot blot analysis. Genomic DNA samples were prepared with twofold serial dilutions in TE buffer and then denatured in $0.4 \mathrm{M} \mathrm{NaOH} / 10 \mathrm{mM}$ EDTA at $95^{\circ} \mathrm{C}$ for $10 \mathrm{~min}$ and followed by adding an equal volume of cold $2 \mathrm{M}$ ammonium acetate ( $\mathrm{pH}$ 7.0). Denatured DNA samples were spotted on a nitrocellulose membrane in an assembled Bio-Dot apparatus (Bio-Rad) according to the manufacturer's instructions. The membrane was washed with $2 \times$ SSC buffer and ultraviolet-crosslinked for $10 \mathrm{~min}$. Then the membrane was blocked with $5 \%$ non-fat milk for $1 \mathrm{~h}$ and incubated with anti-5hmC (Active Motif) or anti-5mC (Active Motif) for HRP-conjugated secondary antibodies and enhanced chemiluminescence detection. The membrane was subsequently stained with methylene blue to confirm corresponding amounts of DNA for each sample. Quantification was performed by ImageJ software analysis.

Chromatin immunoprecipitation assays. Chromatin immunoprecipitation (ChIP) assays were performed as previously described ${ }^{55}$. Briefly, ESCs were crosslinked with $1 \%$ formaldehyde/PBS for $15 \mathrm{~min}$ at room temperature. Crosslinking was quenched by addition of $0.125 \mathrm{M}$ glycine. ESCs were washes twice with ice-cold PBS, and then collected in RIPA buffer as described previously ${ }^{55}$. Samples were then sonicated to generate DNA fragments of approximately $0.5 \mathrm{~kb}$. Approximately, $1 \mu \mathrm{g}$ of pre-cleared protein extract was used for immunoprecipitation with anti-Sirt6 (CST), anti-H3K56ac (Epitomics) or anti-IgG (Abcam) using protein A Sepharose beads (GE Lifesciences) followed by overnight incubation 
at $4^{\circ} \mathrm{C}$. Bead-containing samples were then washed as previously described ${ }^{55}$. Immunocomplexes were eluted by incubation at $65^{\circ} \mathrm{C}$ for $10 \mathrm{~min}$ in the presence of $1 \%$ SDS, and crosslinking was reversed by $5 \mathrm{~h}$ incubation at $65^{\circ} \mathrm{C}$ in the presence of $200 \mathrm{mM} \mathrm{NaCl}$. DNA was purified by the QIAquick spin kit (QIAGEN) and further assessed by qPCR using the LightCycler 480 system from Roche. Data were normalized to input and expressed relative to the nonspecific IgG ChIP control (Fig. 1f) or to the ChIPs from WT samples (Fig. 1g). The primer sequences can be obtained on request.

Teratoma formation assays. Teratomas were obtained by subcutaneous injection of $1 \times 10^{6}$ WT versus S6KO ESCs (C57BL/6) near each quadriceps flank of SCID mice (Charles River Laboratories). Teratomas were collected when the size exceeded $2.0 \mathrm{~cm}$ in diameter and were fixed overnight in $4 \%$ paraformaldehyde. Teratomas derived from S6KO were significantly smaller than WT and they never reached more than $0.5 \mathrm{~cm}$ in diameter. Paraffin sections and staining were performed by the Specialized Rodent Histopathology Core Facility at Harvard Medical School. Animal handling and maintenance were performed in accordance with institutional guidelines. Immunohistochemistry analysis on teratomas was performed by the Histopathology Core Facility at Massachusetts General Hospital.

Chimaera formation. ESCs derived from WT or S6KO (129 mouse strain) were gene targeted with ROSA26-EGFP as described previously ${ }^{56}$ to facilitate tracking of ESC-derived tissues after blastocyst injection. Immunohistochemistry analysis using anti-GFP (Clontech) was performed by the Histopathology Core Facility at Massachusetts General Hospital.

RNA extraction and quantitative PCR with reverse transcription. Total RNA was extracted with the TriPure Isolation Reagent (Roche) as described by the manufacturer. For cDNA synthesis, $1 \mathrm{mg}$ of total RNA was retro-transcribed by using the QuantiTect Reverse Transcription Kit (Qiagen). Real-time PCR was performed using the SYBR green master mix (Roche), following the manufacturer's instructions, with the exception that the final volume was $12.5 \mathrm{ml}$ of SYBR green reaction mix. Real-time monitoring of PCR amplification was performed using the LightCycler 480 detection system (Roche). Data were expressed as relative mRNA levels normalized to the $\beta$-actin expression level in each sample. The primer sequences can be obtained on request.
GEO accession number. The GEO number for the data sets in this manuscript is GSE65836. The GEO numbers for the data sets regarding Sox2 ChIP-Seq are GSM1050291, GSM1050292 and GSM1050293. The GEO number for the human Sirt6 ChIP-Seq data set is GSE32509.

42. Gomes, I. C., Acquarone, M., Maciel Rde, M., Erlich, R. B. \& Rehen, S. K. Analysis of pluripotent stem cells by using cryosections of embryoid bodies. J. Vis. Exp. 46, http://dx.doi.org/10.3791/2344 (2010).

43. Gaspar-Maia, A. et al. Chd1 regulates open chromatin and pluripotency of embryonic stem cells. Nature 460, 863-868 (2013).

44. Shen, $\mathrm{Y}$. et al. A map of the cis-regulatory sequence in the mouse genome. Nature 488, 116-120 (2012).

45. Huang, D. W., Sherman, B. T. \& Lempicki, R. A. Systematic and integrative analysis of large gene lists using DAVID bioinformatics resources. Nat. Protoc. 4, 44-57 (2009).

46. Leinonen, R., Sugawara, H. \& Shumway, M. The sequence read archive. Nucleic Acids Res. 39, 19-21 (2011).

47. Langmead, B., Trapnell, C., Pop, M. \& Salzberg, S. L. Ultrafast and memoryefficient alignment of short DNA sequences to the human genome. Genome Biol. 10, R25 (2009).

48. Li, H. et al. The sequence alignment/Map format and SAMtools. Bioinformatics 25, 2078-2079 (2009).

49. Garber, M. et al. A high-throughput chromatin immunoprecipitation approach reveals principles of dynamic gene regulation in mammals. Mol. Cell 47, 810-822 (2012).

50. Zhang, Y. et al. Model-based analysis of ChIP-Seq (MACS). Genome Biol. 9, R137 (2008).

51. Robinson, J. T. et al. Integrative genomics viewer. Nat. Biotechnol. 29, 24-26 (2011).

52. Bolger, A. M., Lohse, M. \& Usadel, B. Trimmomatic: a flexible trimmer for illumina sequence data. Bioinformatics 30, 2114-2120 (2014).

53. Ying, Q-L., Stavridis, M., Griffiths, D., Li, M. \& Smith, A. Conversion of embryonic stem cells into neuroectodermal precursors in adherent monoculture. Nat. Biotechnol. 21, 183-186 (2003).

54. Sommer, C. A. et al. Induced pluripotent stem cell generation using a single lentiviral stem cell cassette. Stem Cells 27, 543-549 (2009).

55. Gomes, P. N. et al. Gene-specific requirement for P-TEFb activity and RNA polymerase II phosphorylation within the p53 transcriptional program. Genes Dev. 20, 601-612 (2006).

56. Stadtfeld, M. et al. Aberrant silencing of imprinted genes on chromosome 12qF1 in mouse induced pluripotent stem cells. Nature 465, 175-181 (2010). 

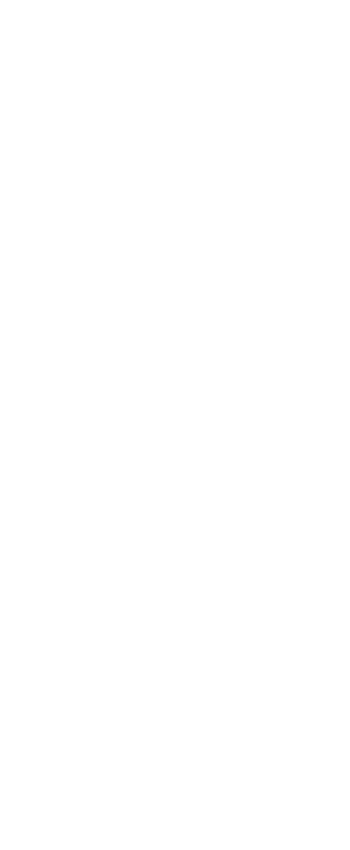

G

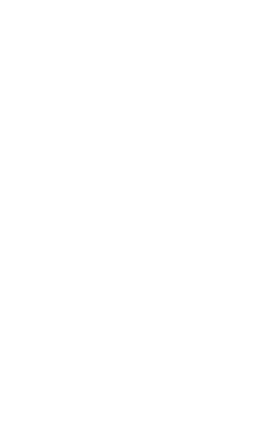

$\mathbf{F}$
C

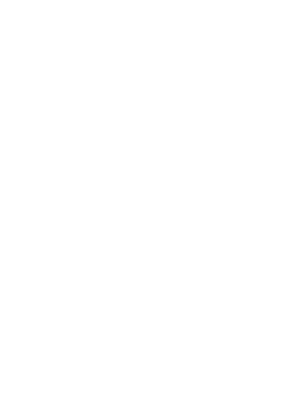

WT

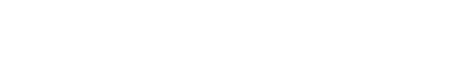

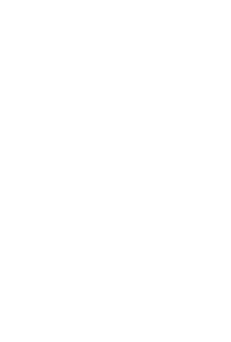

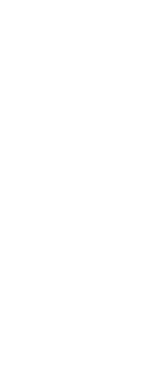
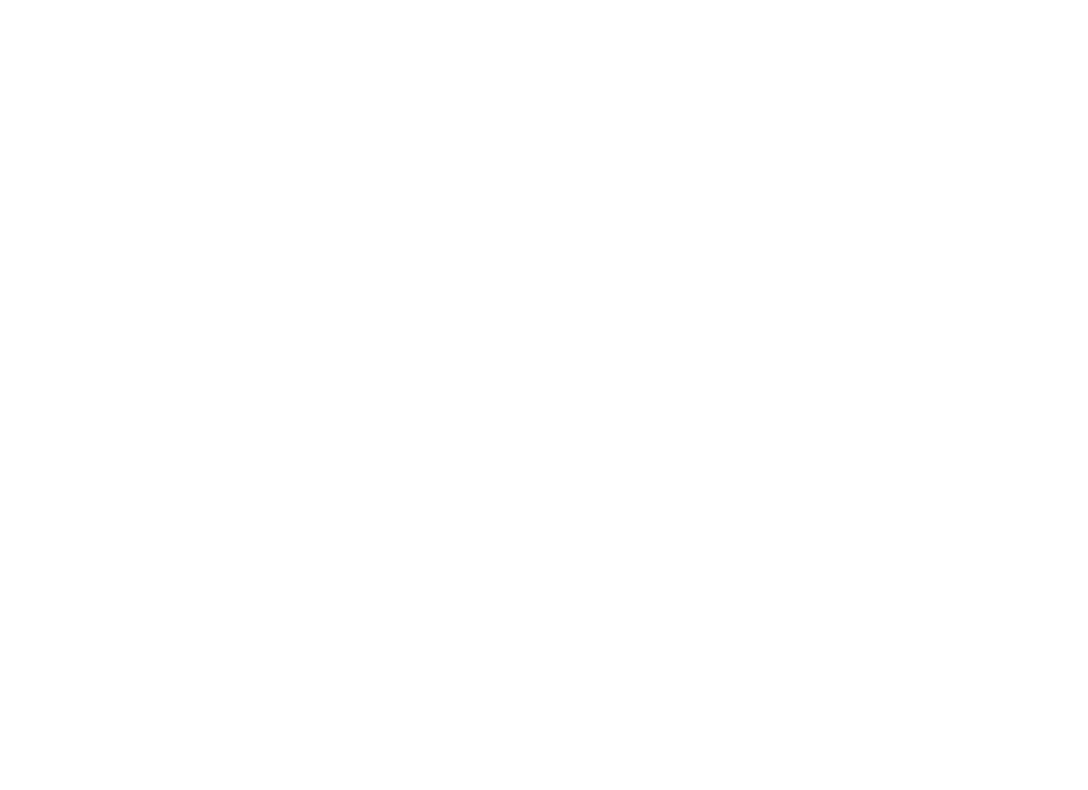

Supplementary Figure 1 Gene expression linked to the skewed phenotype towards neuroectoderm in S6KO EBs, Related to Figure 1. (A) Immunofluorescence of EBs derived from WT and S6KO iPSCs (129 mouse strain) for Gfap (green). Scale bar, $500 \mu \mathrm{m}$. (B) Gene expression of trophectoderm and neuroectoderm genes in WT versus S6KO EBs. qRTPCR data is expressed relative to WT EBs. Data is expressed relative to WT values. The data are $n=3$ experimental replicates (from independent RNA preparations). Values are mean $+/$ - s.e.m. ${ }^{* *} P<0.01,{ }^{* * *} P<0.001,{ }^{* * * * *} P$ $<0.00001$, by $t$-test analysis. (C) Immunofluorescence of in vitro generated neurons from WT and S6KO EBs for $\beta$-III Tubulin (red). Undifferentiated neurons are visualized with dapi staining (blue). This is a representative of $n=3$ experimental replicates (independent experiments). Scale bar, 100 $\mathrm{mm}$.(D) Intensity of immunofluorescence of images from $\mathrm{n}=3$ experimental replicates (independent cell preparations) was analyzed by Image J. Data was normalized to WT values and represented as mean $+/$ - s.e.m. ${ }^{* *} P<0.01$, by $t$-test analysis. (E) Western blots showing expression of Nestin and Tet2 in WT versus S6KO EBs cultured under regular EB-medium. Expression of Sirt6 and its target H3K56ac are also shown. (F) Gene expression of trophectoderm genes in WT versus S6KO ESCs. qRT-PCR data is expressed relative to WT EBs. Data is expressed relative to WT values. The data are $n=3$ experimental replicates (from independent RNA preparations). Values are mean +/- s.e.m. ${ }^{* * *} P<0.001,{ }^{* * * * *} P<0.00001$, by $t$-test analysis. (G) Inability to silence expression of Sox2 and Nanog upon retinoic acid-induced differentiation in S6KO ESCs. Western blot analysis showing the expression of core pluripotent proteins in WT versus S6KO ESCs during a time-course retinoic acid-induced differentiation assay. Molecular weight markers are indicated. (H) Levels of H3K9ac and H3K56ac in S6KO versus WT ESCs after retinoic acid (RA)mediated differentiation. ChIP-Seq binding profiles of the histone marks H3K56ac and H3K9ac on Oct4, Sox2 and Nanog genes in WT versus S6KO ESCs after retinoic acid-mediated differentiation. Images were created with the Integrative Genomic Viewer (IGV) 53 . Data are normalized to total counts, and the scale range is $0.0-1.0$. The data on panels $(B)$ and $(F)$ are $n=3$ experimental replicates, values are mean + - s.e.m. ${ }^{* *} P<0.01,{ }^{* * *} P<$ $0.001,{ }^{* * * * *} P<0.00001$ by $t$-test throughout the figure. 

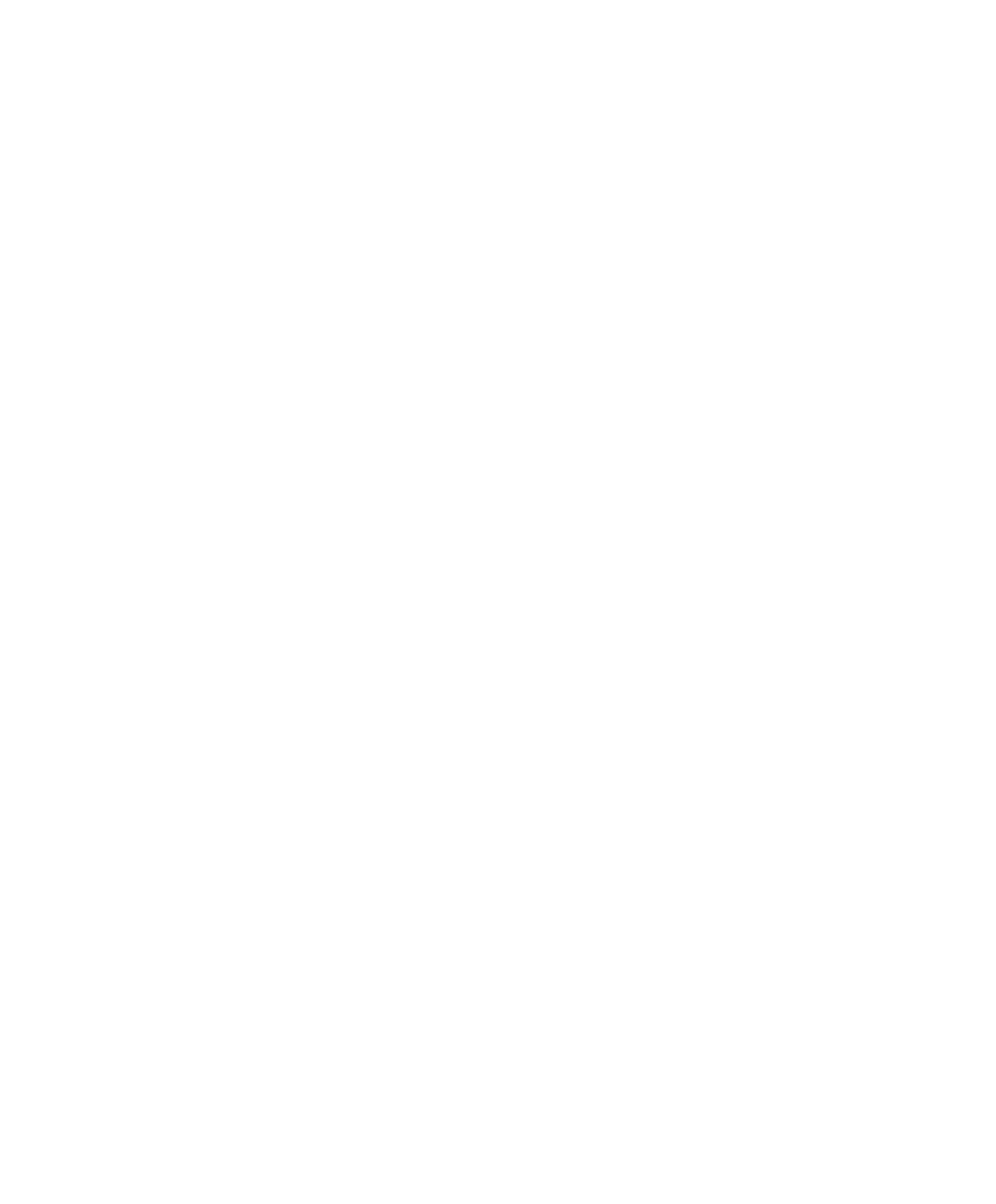

Supplementary Figures 2 and $\mathbf{3}$ The upregulated expression of neuronal differentiation related-genes exhibiting a $5 \mathrm{hmC}$ gain in $\mathrm{S} 6 \mathrm{KO}$ over WT ESCs is rescued upon Tet knockdown, Related to Figures 5 and 6 . Gene expression analysis by qRT-PCR is expressed relative to WT ESCs. Data are $\mathrm{n}=3$ experimental replicates (independent RNA preparations), values are mean + /- s.e.m. ${ }^{*} P<0.05,{ }^{*} P<0.01,{ }^{*} * P<0.001$, $* * * * P<0.0001, * * * * * * * * * P<0.00000001$, by $t$-test throughout the figure. 

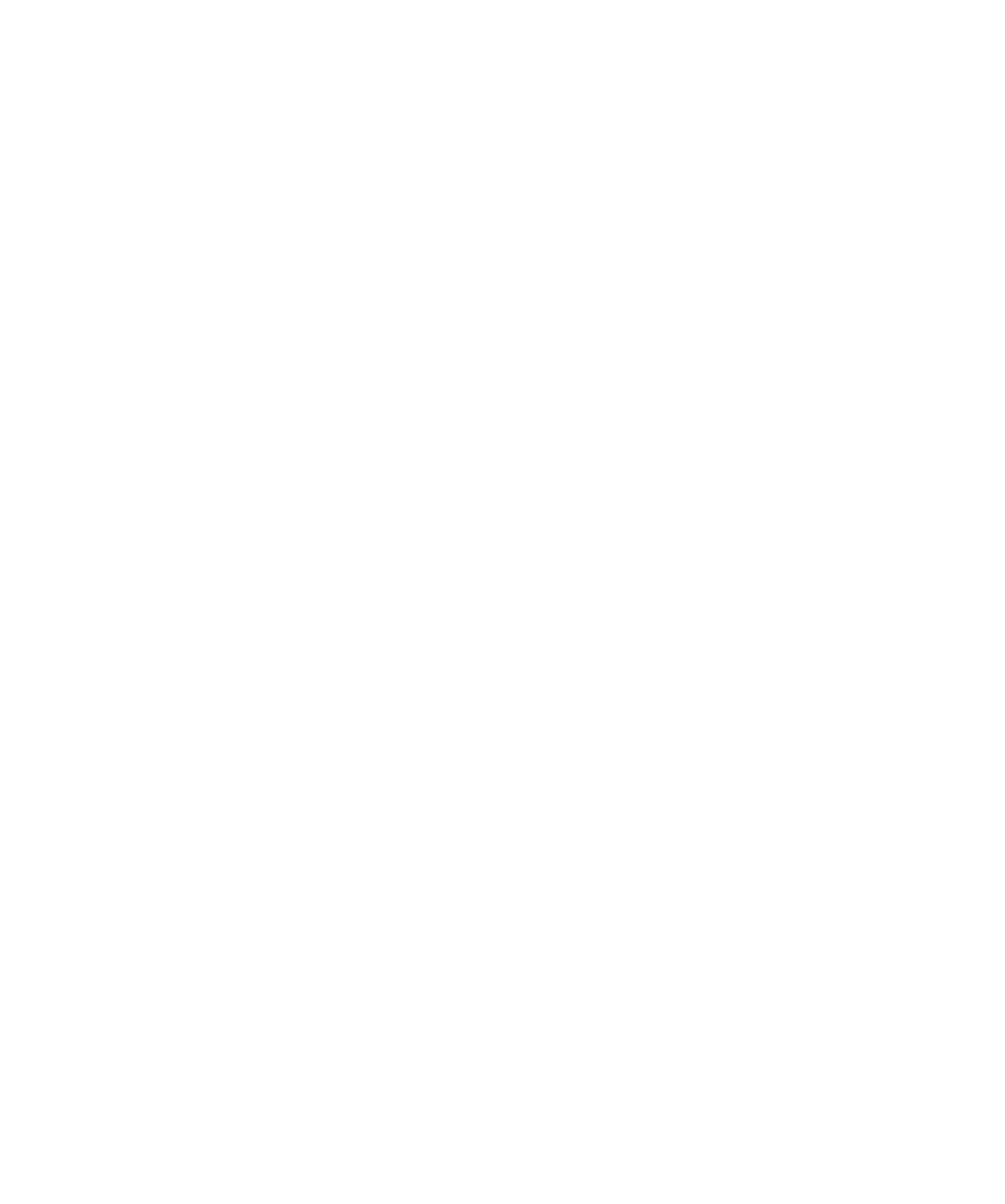

Supplementary Figures 3 The upregulated expression of neuronal differentiation related-genes exhibiting a $5 \mathrm{hmC}$ gain in $\mathrm{S} 6 \mathrm{KO}$ over WT ESCs is rescued upon Tet knockdown, Related to Figures 5 and 6 . Gene expression analysis by qRT-PCR is expressed relative to WT ESCs. Data are $\mathrm{n}=3$ experimental replicates (independent RNA preparations), values are mean + /- s.e.m. ${ }^{*} P<0.05,{ }^{*} P<0.01,{ }^{*} * P<0.001$, $* * * * P<0.0001, * * * * * * * * * P<0.00000001$, by $t$-test throughout the figure. 


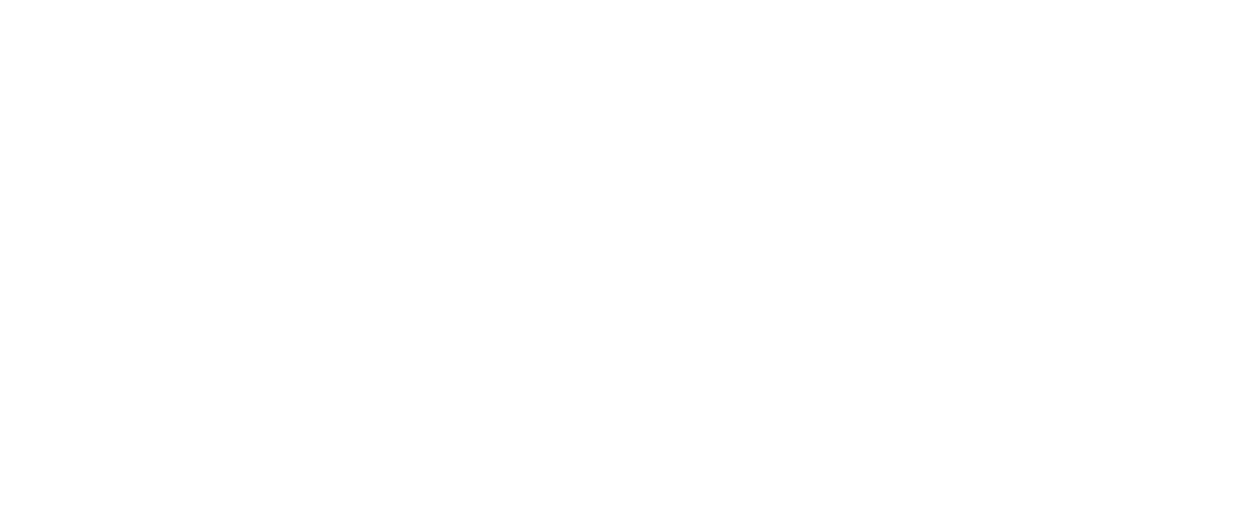

B
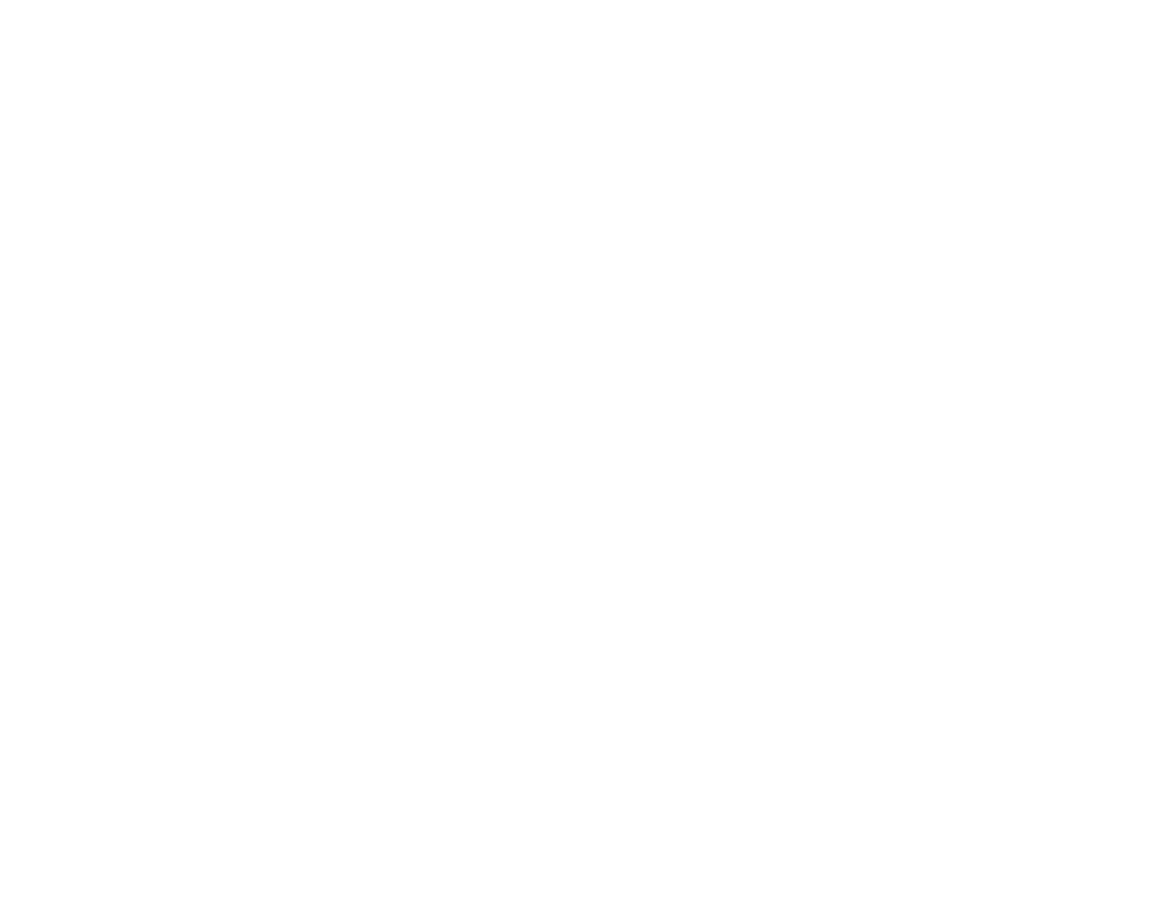

Supplementary Figure 4 (A) The upregulated expression of neuronal differentiation related-genes exhibiting a $5 \mathrm{hmC}$ gain in S6KO over WT ESCs is rescued upon Tet knockdown, Related to Figures 5 and 6 . Gene expression analysis by qRT-PCR is expressed relative to WT ESCs. Data are $\mathrm{n}=3$ experimental replicates (independent RNA preparations), values are mean $+/$ - s.e.m. ${ }^{*} P<0.05,{ }^{* *} P<0.01,{ }^{* * *} P<0.001,{ }^{* * * *} P$ $<0.0001$, by $t$-test throughout the figure. (B) Sirt6 is recruited to the core pluripotent factors in human ESCs, Related to Figure 8. Genome wide Sirt6 ChIP-Seq data originated by Ram and colleagues ${ }^{30}$ was used to determine recruitment of human Sirt6 (hSirt6) to Oct4 and Sox2. (C) A small but significant peak for hSirt6 binding is apparent in Tet1, but not Tet2 gene. Statistically enriched hSirt6 peaks are shown as red boxes. Histone marks (H3K4me1, H3K4me2 and H3K36me3) and p300 signals are also shown for reference. Supplementary Table S10 shows all the genomic regions targeted by hSirt6 analyzed using the same approach as in Ram and colleagues ${ }^{30}$. The accession number for these data can be found at: http://www.ncbi.nlm.nih.gov/geo/query/acc. cgi?acc=GSE32509. 
A
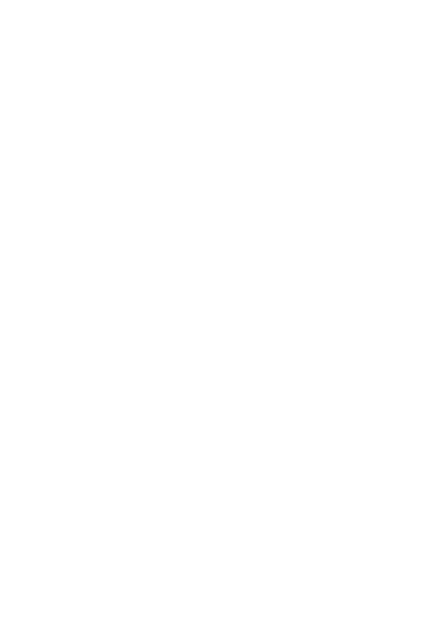

$\mathbf{F}$
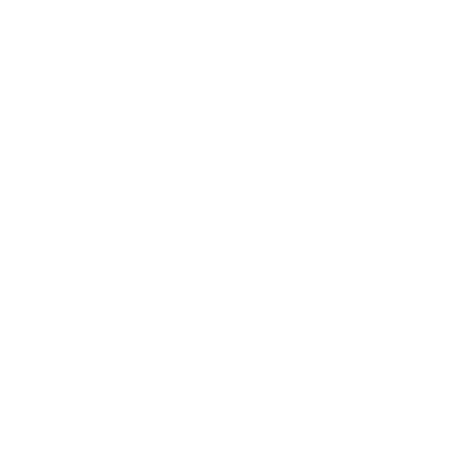

B
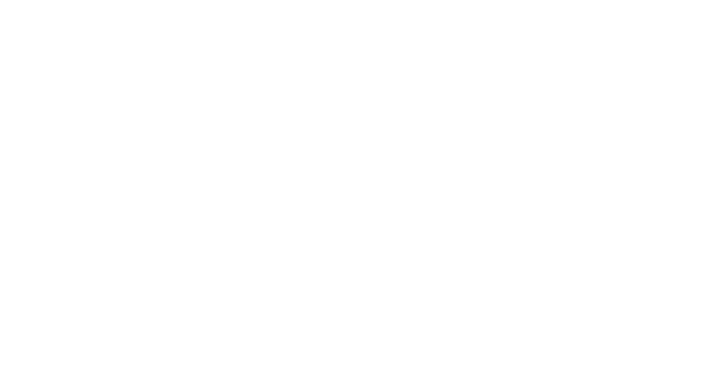

D

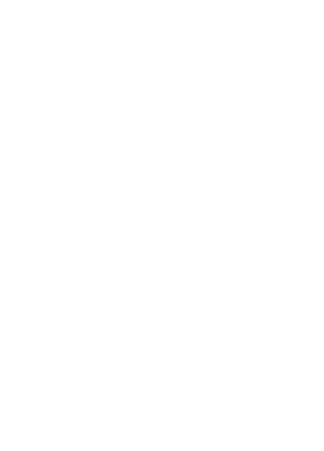

E

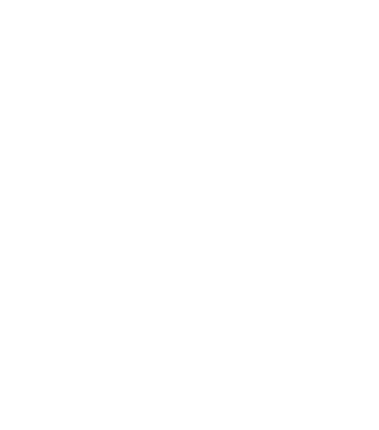

G

H

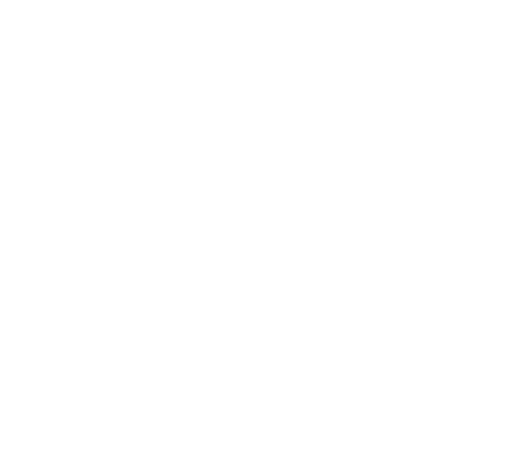

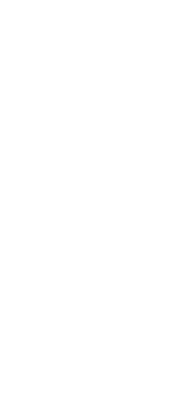

3 商
Supplementary Figure 5 (A) Pdk1 knockdown does not rescue the differentiation phenotype in S6KO EBs, Related to Figure 3. EBs derived from WT and S6KO ESCs (129 mouse strain). Scale bar, $500 \mu \mathrm{m}$. Pictures were taken at days 4 and 6 during EB formation. Data are representative of $n$ $=3$ experimental replicates. (B) Elevated levels of $5 \mathrm{caC}$ in S6KO versus WT ESCs. Related to Figure 4. Global $5 \mathrm{caC}$ levels assayed by slot blot analysis in ESCs. (C) Graph showing densitometric quantification of $5 \mathrm{caC}$ levels at each concentration of genomic DNA from panel (B). Data are representative of $n=$ 2 experimental replicates. (D) Tet downregulation rescues high levels of $5 \mathrm{caC}$ in S6KO versus WT ESCs. Global $5 \mathrm{caC}$ levels assayed by slot blot analysis in Tet knockdown ESCs. (E) Graph showing densitometric quantification of $5 \mathrm{caC}$ levels at each concentration of genomic DNA from panel (D). Data are representative of $n=2$ experimental replicates. (F) Increase efficiency of somatic cell reprogramming in S6KO versus WT NPCs. iPSC colony formation assay measured with alkaline phosphatase. (G) Graph showing iPSC colonies from each genotype. $(H)$ Graph showing the average of WT $(n=3)$ versus S6KO $(n=5)$ NPCs reprogrammed into iPSCs. Colonies were quantified by image $\mathrm{J}$, and values are mean $+/$ - s.e.m. ${ }^{*} P<0.05$, by $t$-test analysis. 

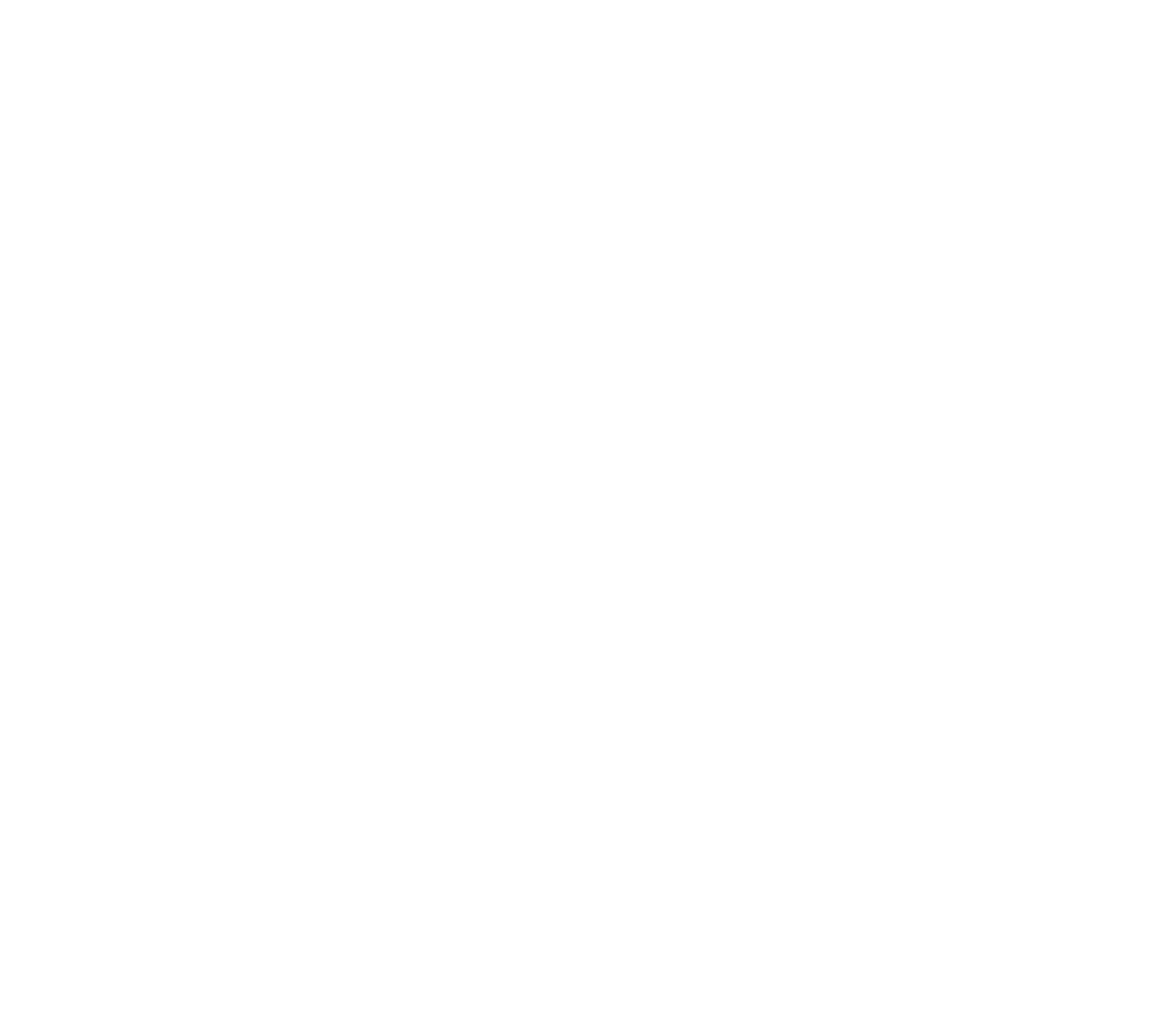

Supplementary Figure 6 Ectopic expression of human Sirt6 rescues the differentiation phenotype of S6KO EBs. (A) Western blot analysis showing expression of endogenous Sirt6 and ectopic hSirt6. Total histone H3 is shown as loading control. (B) Embryoid bodies grown till day 10. Genotypes are indicated. Scale bar, $500 \mathrm{~mm}$. (C) Gene expression analysis by qRT-PCR is expressed relative to WT ESCs. Data are $n=3$ experimental replicates

(independent RNA preparations), values are mean + -- s.e.m. ${ }^{*} P<0.05,{ }^{* *} P$ $<0.01$, by $t$-test analysis. 


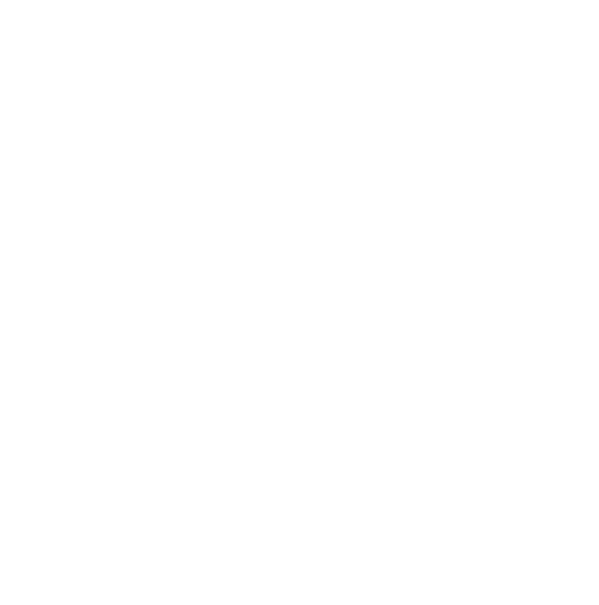

Genomic locations

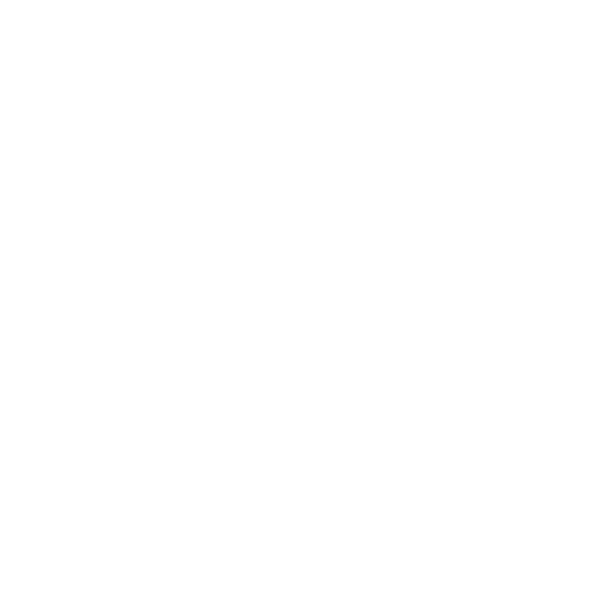

Genomic locations

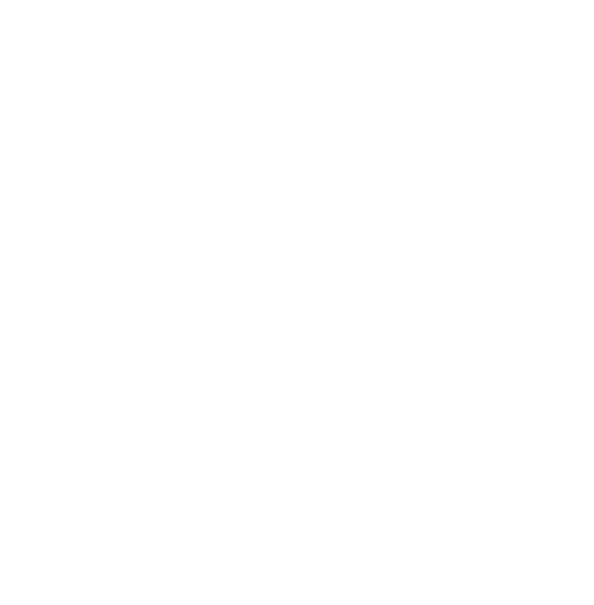

Genomic locations

\section{H3K56ac differential genomic regions in RA-differentiated cells $(P<0.001)$}

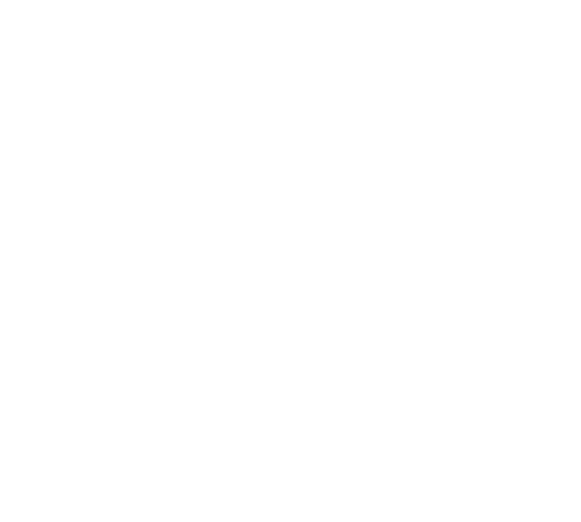

Genomic locations
Supplementary Figure 7 Characterization of genomic regions with changes of H3K9ac and H3K56ac in S6KO versus WT ESCs, before and after retinoic acid (RA)-mediated differentiation. MEDIPS software was used to find regions with differential histones $\mathrm{H} 3 \mathrm{~K} 9 \mathrm{ac}$ and $\mathrm{H} 3 \mathrm{~K} 56 \mathrm{ac}$ in 500 base windows with $p<0.001$ and these regions with gains and losses upon Sirt6 KO were mapped to promoters, gene bodies,
CpG islands, enhancers, and super-enhancers.(A) Genomic positions of H3K9ac gains and losses in mouse ESCs. (B) Genomic positions of H3K9ac gains and losses in RA-differentiated mouse ESCs. (C) Genomic positions of H3K56ac gains and losses in mouse ESCs. (D) Genomic positions of H3K56ac gains and losses in RA-differentiated mouse ESCs. 
A

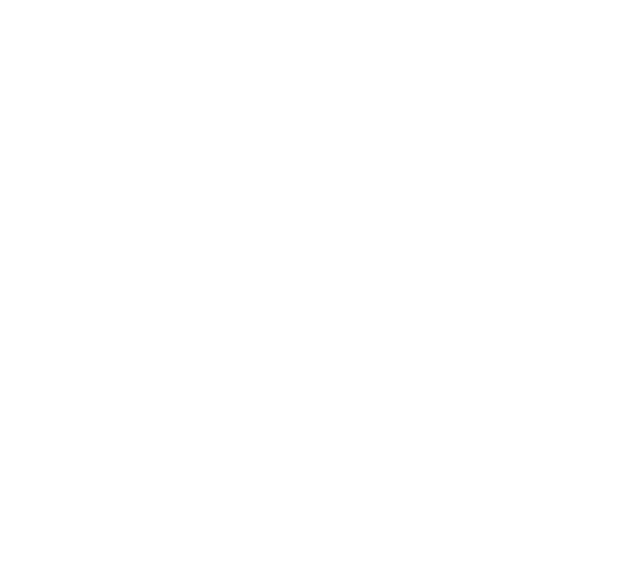

C

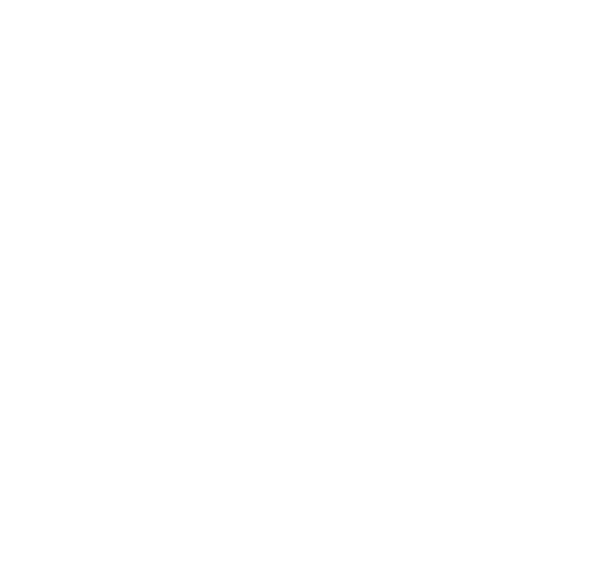

B

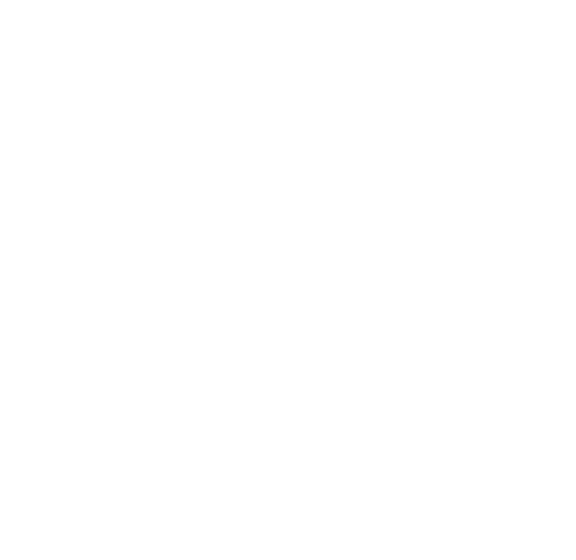

D

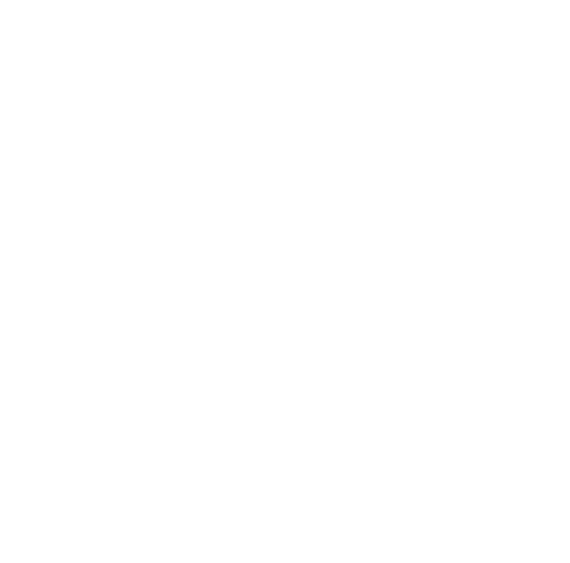

Supplementary Figure 8 Enrichment of $5 \mathrm{hmC}$ does not correlate with $\mathrm{H} 3 \mathrm{~K} 9 \mathrm{ac}$ and/or H3K56ac in S6KO versus WT ESCs. (A) Heat map plot of regions of H3K56ac gains found with MEDIPS software in S6KO over WT in mouse ESCs show average profile for a $+/-3 \mathrm{~kb}$ band centered around the 18722 regions with $\mathrm{H} 3 \mathrm{~K} 56 \mathrm{ac}$ gain $(\mathrm{p}<0.001)$ for the factors $5 \mathrm{hmC}, \mathrm{H} 3 \mathrm{~K} 56 \mathrm{ac}$, H3K9ac, Sirt6, and Sox2 in WT and S6KO ESCs. (B) Enrichment line plot of average profile for regions of H3K56ac gain in S6KO over WT in mouse ESCs ( $n=18722)$ for the data on panel (A). Semi-transparent band behind line shows standard error of the mean for each average profile. (C) Heat map plot of regions of H3K9ac gains found with MEDIPS software in S6KO over WT mouse ESCs show average profile for a $+/-3 \mathrm{~kb}$ band centered around the 39095 regions with $\mathrm{H} 3 \mathrm{~K} 9 \mathrm{ac}$ gain $(\mathrm{p}<0.001)$ for the factors $5 \mathrm{hmC}, \mathrm{H} 3 \mathrm{~K} 56 \mathrm{ac}$, H3K9ac, Sirt6, and Sox2 in WT and S6KO ESCs. (D) Enrichment line plot of average profile for regions of H3K9ac gain in S6KO over WT in mouse ES cells ( $n=39095)$ for the data on panel (C). Semi-transparent band behind line shows standard error of the mean for each average profile. 


\section{Related to Figure 11}

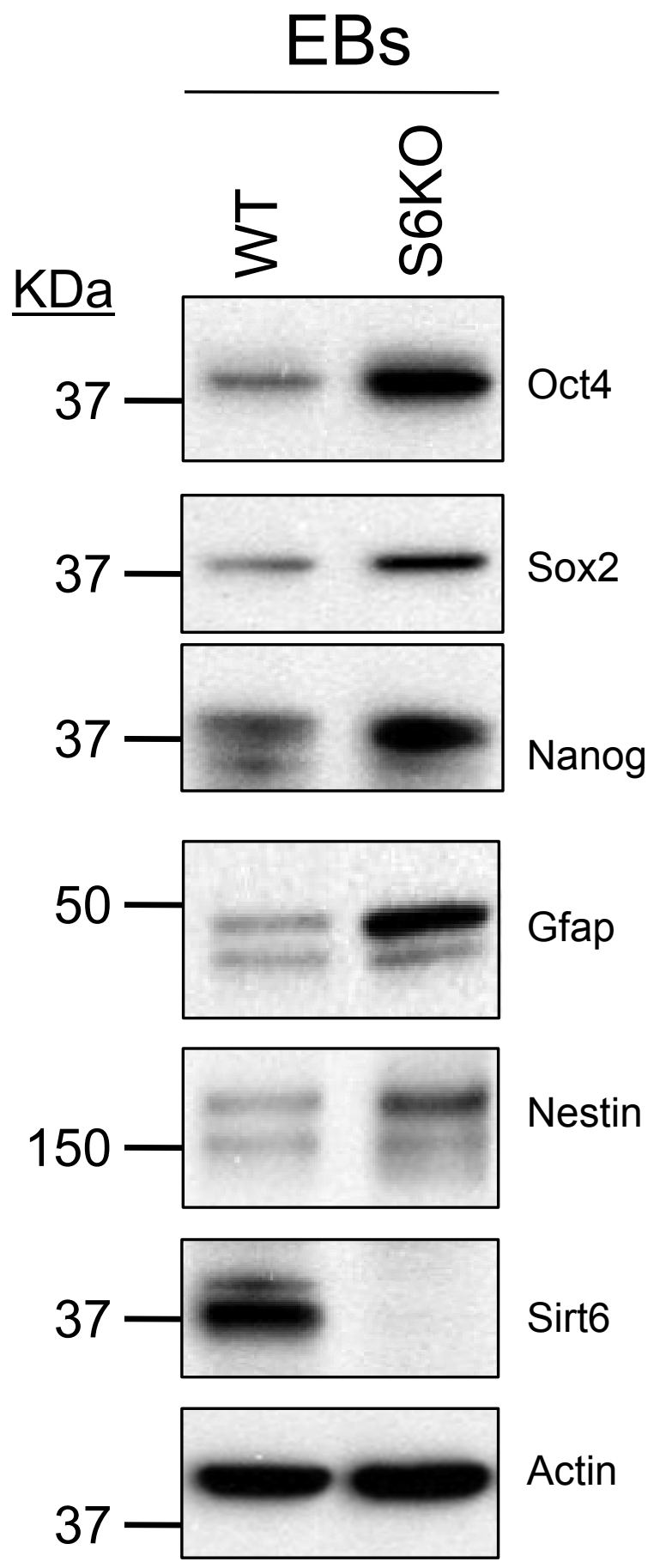

Supplementary Figure 9 This is a copy of Figure 1I, which is based on Supplementary Figures (B), (C) and (D). These are unprocessed scanned Western blot panels showing a time course (days 3,6 and 10) differentiation of EB formation. Molecular weight (KDa) ladders are shown for each Western blot panel. 
Related to Figure 11

EBs
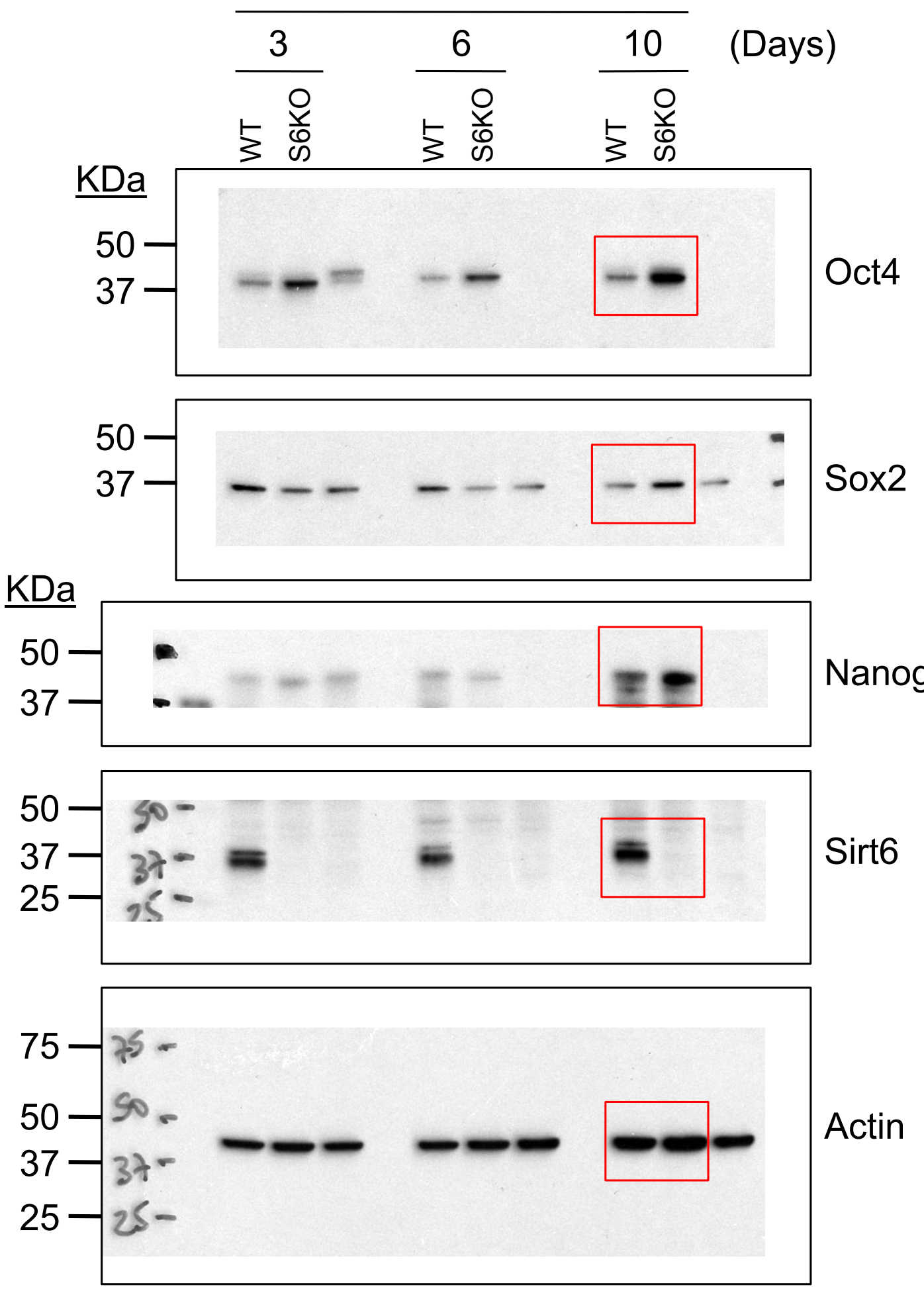

Supplementary Figure 9 continued The red boxes highlight the expression of Oct4, Sox2, Nanog, Sirt6 and Actin (as loading control), as shown in Figure 1 . 
Related to Figure 11
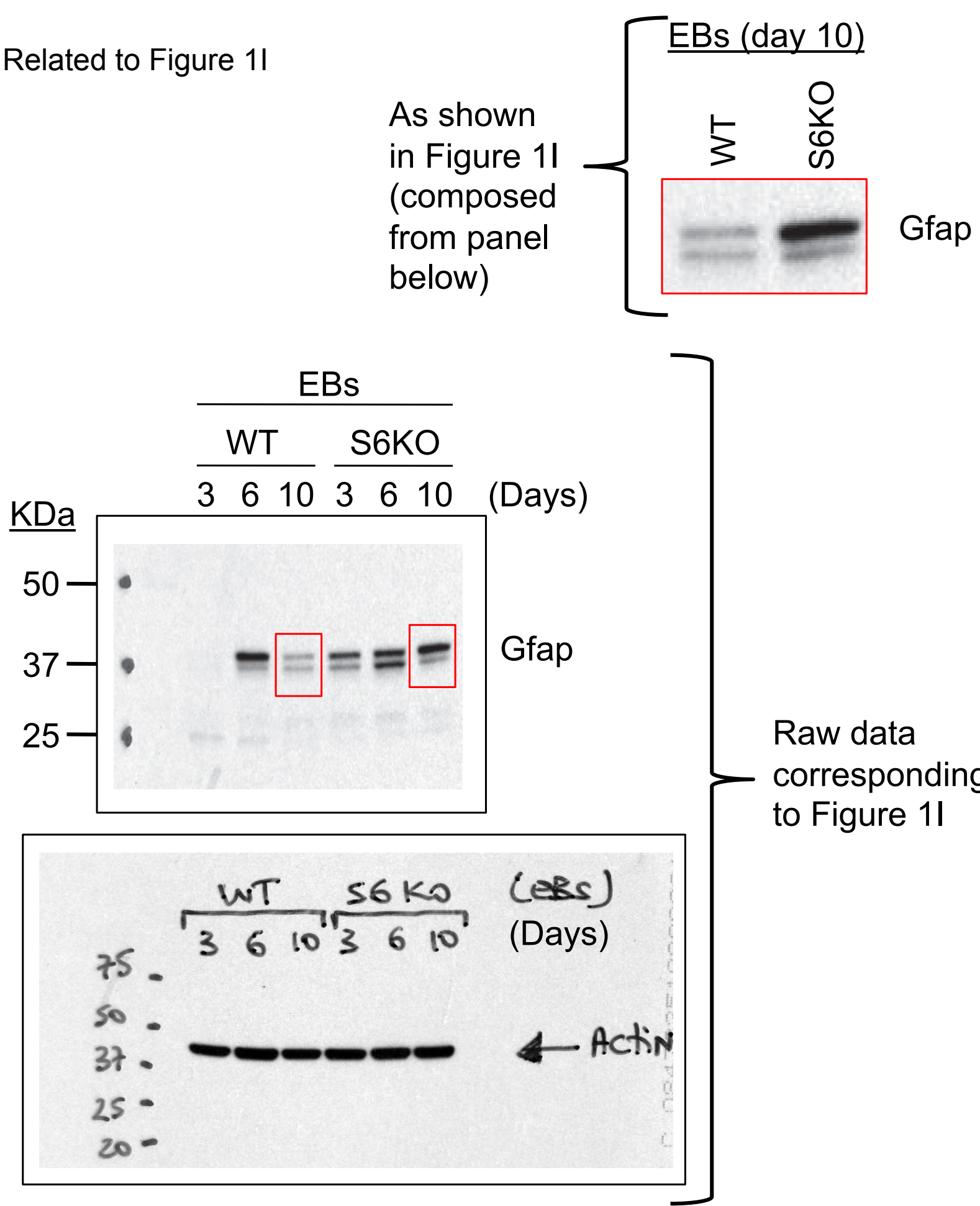

Raw data

corresponding to Figure 11 


\section{Related to Figure 11}
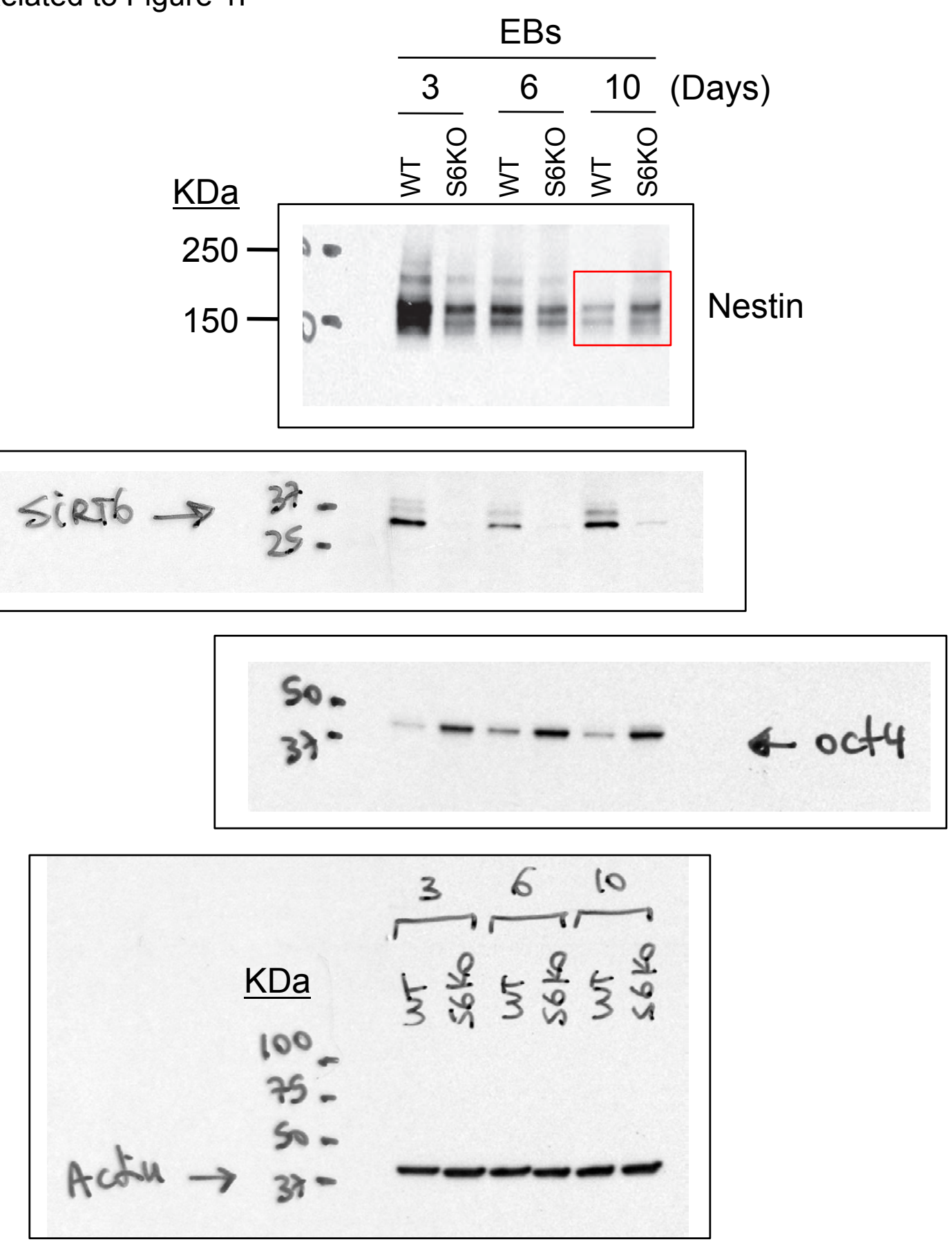

Supplementary Figure 9 continued The red box highlights the expression of Nestin as shown in Figure 1I. Expression of Sirt6, Oct4 and Actin (as loading control) are included, since these are distinct Western blots from panel (B). 
ESCs
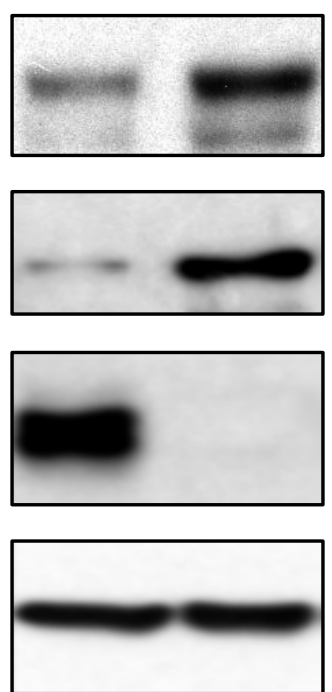

$\sum$
$\frac{\vartheta}{0}$

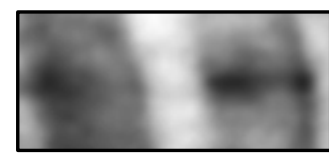

\section{Tet1}

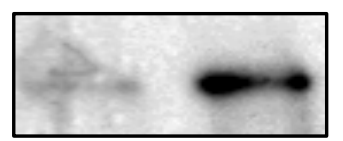

Tet2

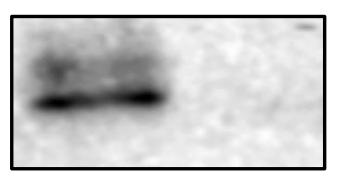

Sirt6

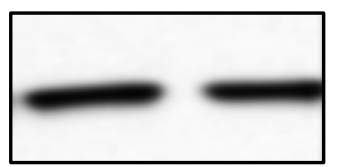

\section{Actin}

$\sum \quad \frac{\vartheta}{\omega}$

Supplementary Figure 9 continued This is a copy of Figure 3B, which is based on Supplementary Figures (F), (G) and (H). These are unprocessed scanned Western blot panels showing protein expression from ESCs and EBs along with molecular weight (KDa) ladders. 
Related to Figure 3B

\section{ESCs}
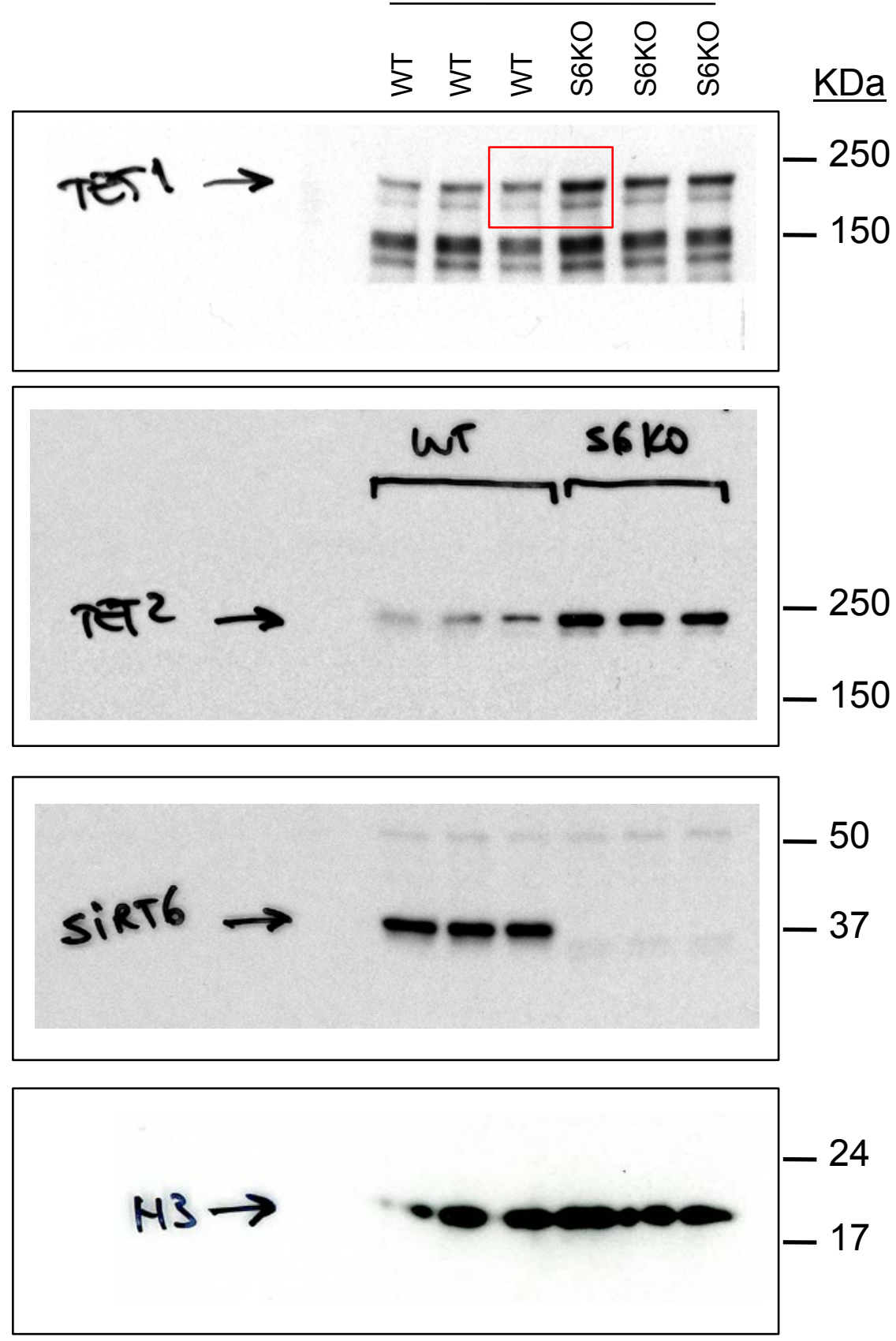

Supplementary Figure 9 continued The red box in the upper panel shows expression of Tet1 as shown in Figure 3B. Expression of Tet2 and Sirt6 are also included. Histone $\mathrm{H} 3$ was used as loading control. 
Related to Figure 3B

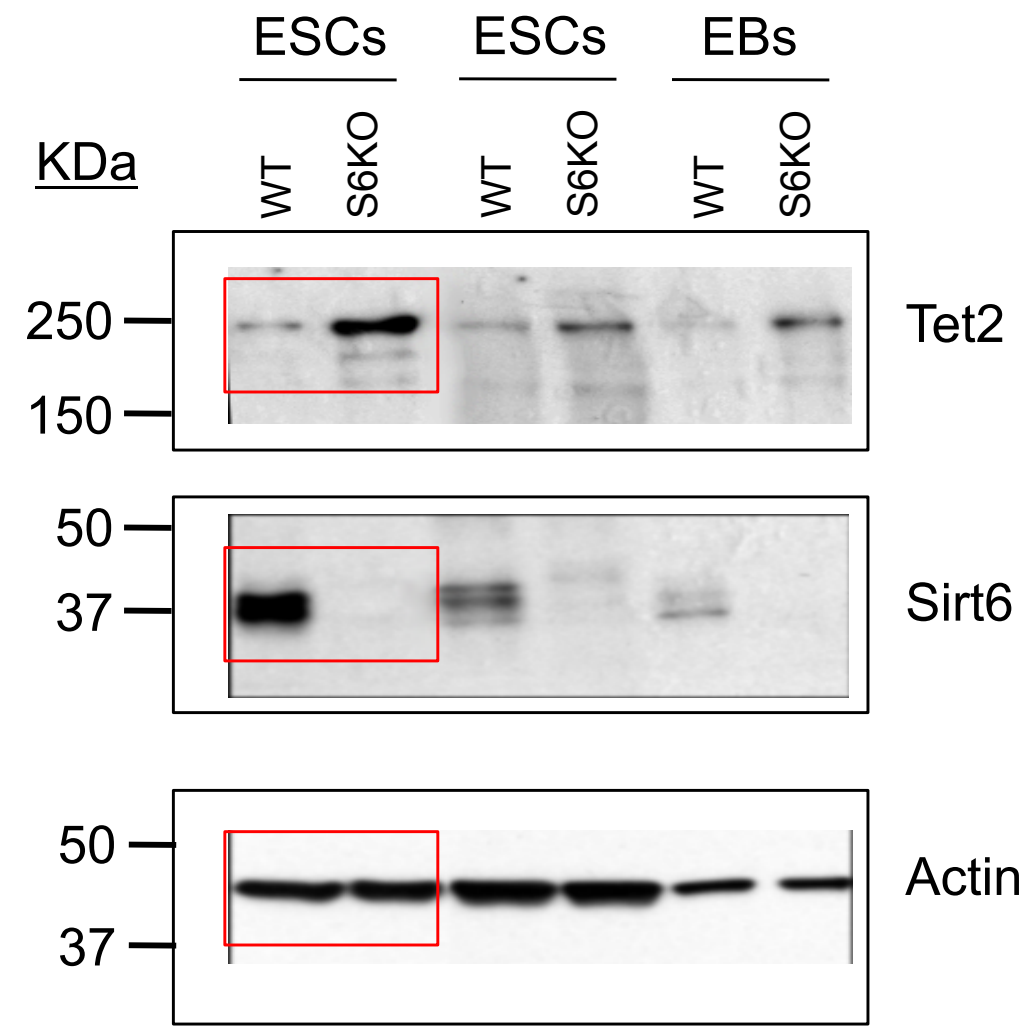

Supplementary Figure 9 continued The red boxes highlight the expression of Tet2, Sirt6 and Actin in ESCs, as shown in Figure 3B. 

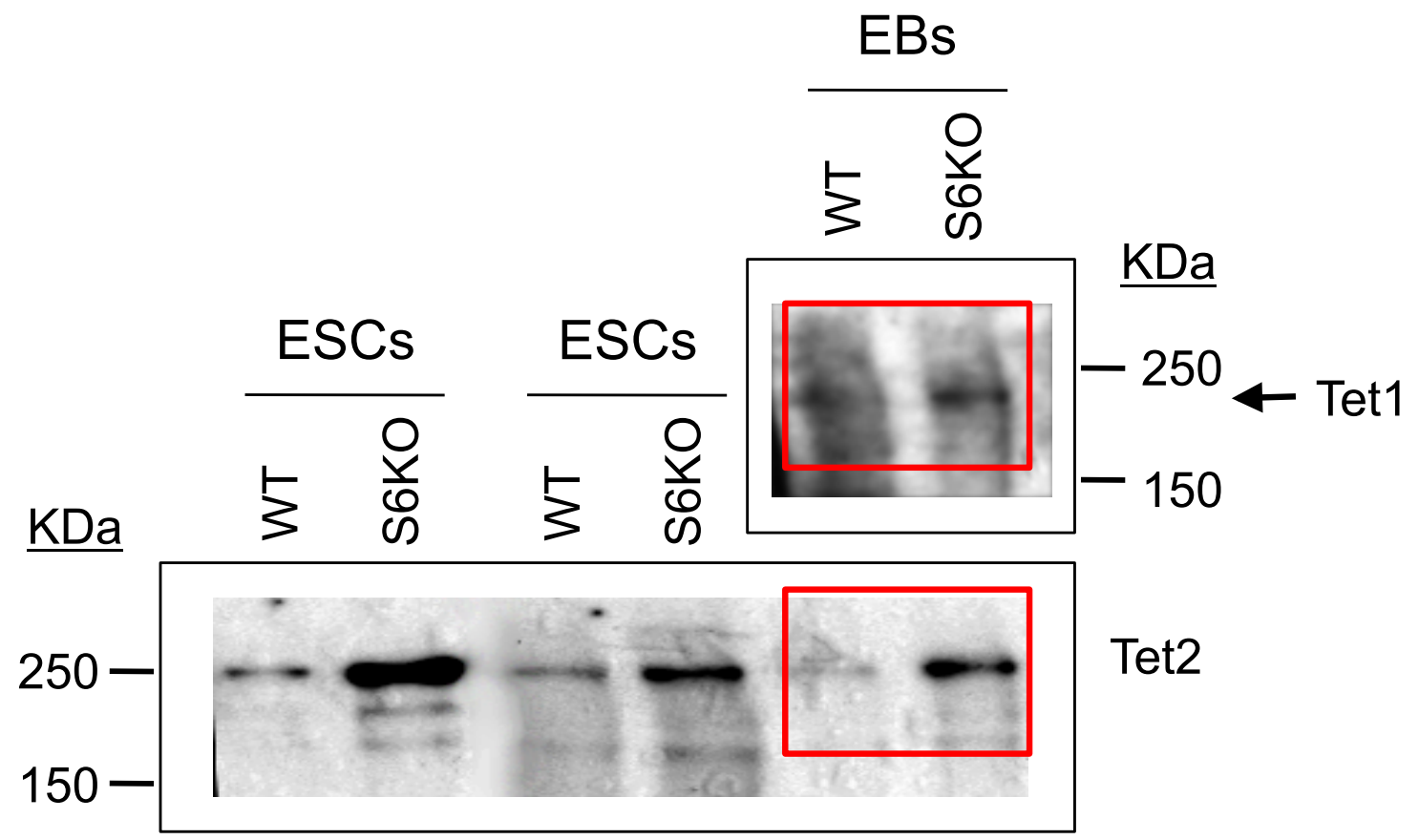

Tet2

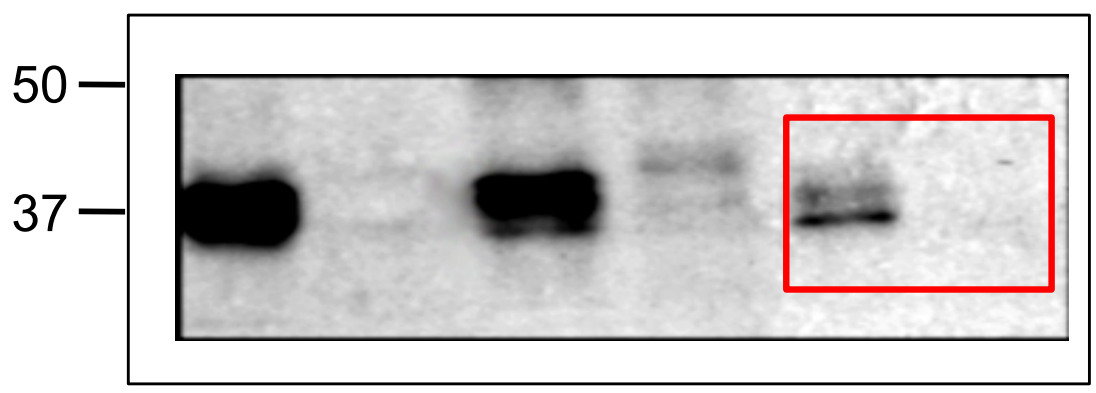

Sirt6

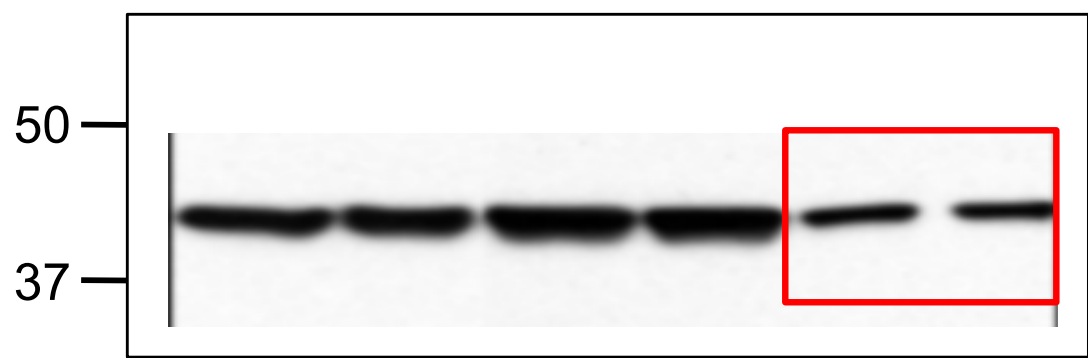

Actin

Supplementary Figure 9 continued The red boxes highlight the expression of Tet1, Tet2, Sirt6 and Actin in EBs, as shown in Figure 3B. 


\section{Supplementary Table Legends}

Supplementary Table 1 Identification of differentially hydroxymethylated regions (DHMRs).

Supplementary Table 2 Gene ontology annotation of DHMRs.

Supplementary Table 3 Enrichment of histone modifications and transcription factor binding.

Supplementary Table 4 Lists of genes with Sirt6 peaks associated with promoters (TSS $+/-1 \mathrm{~kb}$ ) and exons in mouse ESCs. List of mouse ESC gene markers with Sirt6 peaks associated with transcription start sites (TSS), exons and introns in mouse ESCs.

Supplementary Table 5 Gene lists with differential H3K56ac (gain or loss of H3K56ac in S6KO compared to WT) at promoters (TSS +/- 1kb) or exons in mouse ESCs.

Supplementary Table 6 Genes lists with differential H3K9ac (gain or loss of H3K9ac in S6KO compared to WT) at promoters (TSS +/- 1kb) or exons in untreated mouse ESCs. A second list of promoter gain with $p<1 \mathrm{e}-7$ and log fold change $>4$ was included because DAVID analysis is limited to lists of 3000 or fewer genes.

Supplementary Table 7 Genes lists with differential H3K56ac (gain or loss of H3K56ac in S6KO compared to WT) at promoters (TSS +/- 1kb) or exons in mouse ESCs treated with differentiating agent, retinoic acid (RA).

Supplementary Table 8 Genes lists with differential H3K9ac (gain or loss of H3K9ac in S6KO compared to WT) at promoters (TSS +/- 1kb) or exons in mouse ESCs treated with the differentiating agent, retinoic acid (RA).

Supplementary Table 9 Lists of genes with Sox 2 peaks associated with promoters (TSS $+/-1 \mathrm{~kb}$ ) and exons in mouse ESCs. Data is from Lodato and colleagues $^{29}$ using GEO sample GSM1050291 or SR1050291 along with corresponding whole cell extract (WCE) controls in standard ChIP-Seq pipeline with MAC2 for peak detection.

Supplementary Table 10 List of 2,604 genomic regions ("peaks") called for Sirt6 using the same approach employed in Ram and colleagues 30 .

Supplementary Table S11 Mendelian frequencies calculated from $n=35$ mouse litters (total of 223 born pups). 